\title{
Tight Frames and their Symmetries
}

\author{
Richard Vale, Shayne Waldron \\ Department of Mathematics, University of Auckland, Private Bag 92019, Auckland, New Zealand \\ e-mail: waldron@math.auckland.ac.nz (http:www.math.auckland.ac.nz/ ${ }^{\sim}$ waldron) \\ e-mail: vale@math.auckland.ac.nz
}

\begin{abstract}
The aim of this paper is to investigate symmetry properties of tight frames, with a view to constructing tight frames of orthogonal polynomials in several variables which share the symmetries of the weight function, and other similar applications. This is achieved by using representation theory to give methods for constructing tight frames as orbits of groups of unitary transformations acting on a given finite-dimensional Hilbert space. Along the way, we show that a tight frame is determined by its Gram matrix and discuss how the symmetries of a tight frame are related to its Gram matrix. We also give a complete classification of those tight frames which arise as orbits of an abelian group of symmetries.
\end{abstract}

Key Words: Tight frames, isometric tight frames, Gram matrix, multivariate orthogonal polynomials, symmetry groups, harmonic frames, representation theory, wavelets

AMS (MOS) Subject Classifications: primary 05B20, 33C50, 20C15, 42C15, secondary 52B15, 42C40 


\section{Introduction}

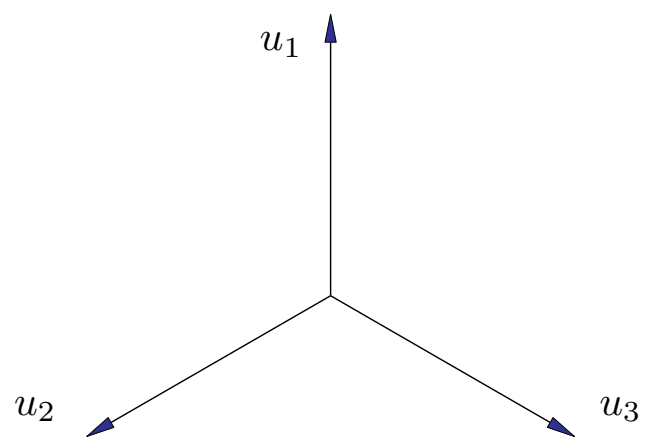

The three equally spaced unit vectors $u_{1}, u_{2}, u_{3}$ in $\mathbb{R}^{2}$ provide the following redundant representation

$$
f=\frac{2}{3} \sum_{j=1}^{3}\left\langle f, u_{j}\right\rangle u_{j}, \quad \forall f \in \mathbb{R}^{2},
$$

which is the simplest example of a tight frame. Such representations arose in the study of nonharmonic Fourier series in $L_{2}(\mathbb{R})$ (see Duffin and Schaeffer [DS52]) and have recently been used extensively in the theory of wavelets (see, e.g., Daubechies [D92]).

The philosophy behind the use of frames is that representations such as (1.1) are similar to an orthogonal expansion (but with more terms), and that by going to a frame representation one might choose the $u_{j}$ to have desirable properties that may be impossible were they to be orthogonal (in the case of wavelets these are certain smoothness and small support properties).

In particular, a tight frame may be preferred to an orthogonal basis if there are underlying symmetries which it is desirable for the tight frame to have, but which cannot be possessed by any orthogonal basis. We are particularly interested in spaces of orthogonal polynomials of several variables with weights with some symmetries, e.g., integration over a polyhedron in $\mathbb{R}^{2}$. Here we hope to replace orthonormal bases with spanning sets that share symmetries of the weight and are tight frames.

In this paper, we set out to find ways of constructing tight frames which are invariant under a given group of symmetries of some finite-dimensional Hilbert space $\mathcal{H}$. The paper is organised as follows. In Section 2, we define tight frames and give some of their elementary properties. In particular, we show that a spanning set is determined up to a unitary transformation by the inner products of its elements, a useful result which we have not found elsewhere (Theorem 2.9). In Section 3, we define the key notion of symmetries of a tight frame. In Section 4, we investigate how the symmetries of a tight frame are reflected in the structure of its Gram matrix (Theorem 4.2). In Section 5, we investigate tight frames generated by abelian groups of unitary transformations, and classify all such frames (Theorem 5.4). There has been some interest in frames of this type, see eg. [BE03]. In Section 6, we use representation theory to show how to construct tight frames which arise as orbits of an arbitrary group $G$ of unitary transformations. The situation is particularly simple if every orbit of $G$ spans $\mathcal{H}$. In this case, every $G$-orbit is a tight frame (Theorem 
6.3). Theorem 6.4 says that most of the examples constructed in this way cannot be obtained as an orbit of an abelian group. In Section 7, we exploit the results of Section 6 to construct some new highly symmetric tight frames of orthogonal polynomials of low degree on a triangle, square and hexagon, including some orthonormal bases sharing symmetries, which were not previously known to exist.

\section{Finite tight frames}

Let $\mathcal{H}$ be a $d$-dimensional real or complex Hilbert space.

Definition 2.1. A finite set $\Phi=\left\{\phi_{j}\right\}_{j=1}^{n}$ of nonzero vectors in $\mathcal{H}$ is a tight frame for $\mathcal{H}$ if there exists $c>0$ with

$$
\|f\|^{2}=c \sum_{j=1}^{n}\left|\left\langle f, \phi_{j}\right\rangle\right|^{2}, \quad \forall f \in \mathcal{H}
$$

By the polarisation identity, (2.2) is equivalent to the (possibly redundant) representation

$$
f=c \sum_{j=1}^{n}\left\langle f, \phi_{j}\right\rangle \phi_{j}=\sum_{j=1}^{n}\left\langle f, \sqrt{c} \phi_{j}\right\rangle \sqrt{c} \phi_{j}, \quad \forall f \in \mathcal{H} .
$$

We will refer to $\left\{\sqrt{c} \phi_{j}\right\}$ as the standard form of $\Phi$, and identify all tight frames with the same standard form. We say a tight frame $\Phi$ is isometric if all $\phi \in \Phi$ have equal norm (the term normalized tight frame is also in use, cf [Z01]).

Every finite-dimensional tight frame of cardinality $n$ for a $d$-dimensional space can be thought of as the projection of an orthonormal basis for an $n$-dimensional space onto a suitably chosen $d$-dimensional subspace (cf Naimark's theorem in [AG93]).

Example 1. For $n \geq 3$, define $\Phi \subset \mathbb{R}^{2}$ by

$$
\phi_{k}=\left[\begin{array}{c}
\cos (2 \pi k / n) \\
\sin (2 \pi k / n)
\end{array}\right], \quad k=1, \ldots n
$$

Then $\Phi=\left\{\phi_{k}\right\}_{k=1}^{n}$ is an isometric tight frame for $\mathbb{R}^{2}$ called the $n^{\text {th }}$ roots of unity.

Example 2. Let $\left\{e_{1}, \ldots, e_{d+1}\right\}$ be an orthonormal basis for $\mathbb{R}^{d+1}$, and $z:=\sum_{i=1}^{d+1} e_{i}$. Then the orthogonal projection of $\left\{e_{i}\right\}$ onto the orthogonal complement of $z$ (which we identify with $\mathbb{R}^{d}$ ) is an isometric tight frame for $\mathbb{R}^{d}$ which we call the regular $d$-simplex. The distances between any two elements of this isometric tight frame are equal.

Applying a unitary transformation $U$ on $\mathcal{H}$ to $(2.3)$ gives

$$
U f=c \sum_{\phi \in \Phi}\langle f, \phi\rangle U \phi=c \sum_{\phi \in \Phi}\langle U f, U \phi\rangle U \phi, \quad \forall f \quad \Longrightarrow \quad f=c \sum_{\phi \in \Phi}\langle f, U \phi\rangle U \phi, \quad \forall f
$$

so that a unitary image of a tight frame $\Phi$ is again a tight frame (with the same scaling factor $c$ ), which we consider to be equivalent by the following equivalence relation. 
Definition 2.4. Tight frames $\Phi$ and $\Psi$ in standard form $(c=1)$ are said to be equivalent if there exists a unitary $U$ for which

$$
U \Phi=\Psi
$$

For $\Phi=\left\{\phi_{i}\right\}_{i=1}^{n}, \Psi=\left\{\psi_{j}\right\}_{j=1}^{m},(2.5)$ means there is a permutation $\pi$ of the indices with

$$
\psi_{\pi j}=U \phi_{j}
$$

This equivalence preserves many properties we are interested in, most importantly the inner products between vectors and the symmetries of a frame (to be defined later).

There is another notion of equivalence of frames found in [GKK01] and [F01], where $\psi_{\pi j}=U \phi_{j}$ in (2.6) is replaced by $\psi_{\pi j}=\lambda_{j} U \phi_{j}$ for some scalar $\lambda_{j}$ of unit modulus. This equivalence does not preserve the inner product between vectors.

Example 3. There are many examples of inequivalent tight frames - even isometric ones. For example, take three equally spaced vectors in $\mathbb{R}^{2}$ (which form an isometric tight frame) together with the equivalent tight frame obtained by rotating them through $0<\theta<2 \pi / 3$. The resulting set of six vectors is an isometric tight frame for $\mathbb{R}^{2}$. For different values of $\theta$ such tight frames are clearly not equivalent, since unitary transformations preserve angles.
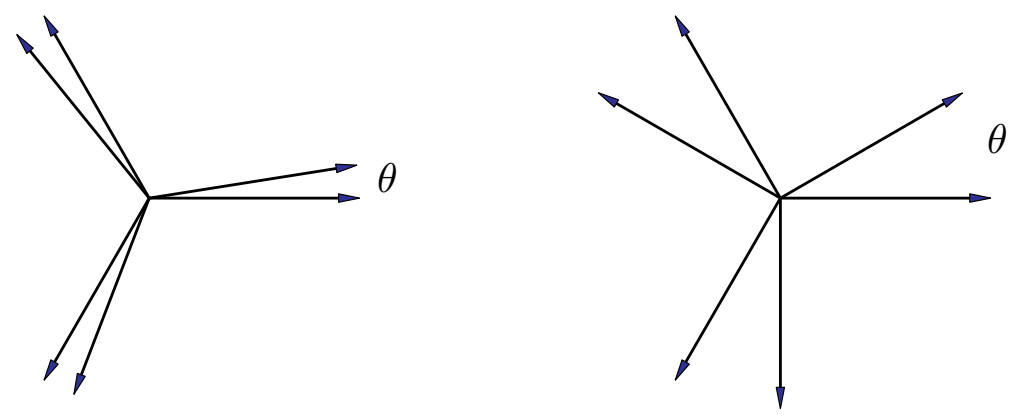

We now show that frames are equivalent if and only if the inner products between their vectors are the same. The Gram matrix or Gramian of a sequence of vectors $\Phi=\left(\phi_{1}, \ldots, \phi_{n}\right)$ is the $n \times n$ matrix

$$
\operatorname{Gram}(\Phi):=\left[\left\langle\phi_{i}, \phi_{j}\right\rangle\right]_{i, j=1}^{n} .
$$

Lemma 2.7. Suppose $\Phi=\left(\phi_{1}, \ldots \phi_{n}\right), \Psi=\left(\psi_{1}, \ldots \psi_{n}\right)$ are sequences of vectors, and that $\operatorname{span}(\Phi)=\mathcal{H}$. Then there exists a unitary $U$ with $U \phi_{i}=\psi_{i}, \forall i$ if and only if

$$
\left\langle\phi_{i}, \phi_{j}\right\rangle=\left\langle\psi_{i}, \psi_{j}\right\rangle, \quad \forall i, j,
$$

i.e., the Gram matrices of $\Phi$ and $\Psi$ are equal.

Proof: If there is such a $U$, then clearly $\left\langle\psi_{i}, \psi_{j}\right\rangle=\left\langle U \phi_{i}, U \phi_{j}\right\rangle=\left\langle\phi_{i}, \phi_{j}\right\rangle, \forall i, j$. 
Now suppose (2.8) holds. Assume without loss of generality that $\left\{\phi_{1}, \ldots \phi_{d}\right\}$ is a basis for $\mathcal{H}$, and define a linear map $U$ on $\mathcal{H}$ by $U \phi_{i}=\psi_{i}, 1 \leq i \leq d$. Then $U$ is unitary, since for $f=\sum_{i=1}^{d} \lambda_{i} \phi_{i}, g=\sum_{j=1}^{d} \mu_{j} \phi_{j}$, we have

$$
\langle U f, U g\rangle=\sum_{i, j} \lambda_{i} \overline{\mu_{j}}\left\langle\psi_{i}, \psi_{j}\right\rangle=\sum_{i, j} \lambda_{i} \overline{\mu_{j}}\left\langle\phi_{i}, \phi_{j}\right\rangle=\langle f, g\rangle .
$$

For $i>d$ and $1 \leq j \leq d$, we have

$$
\left\langle U \phi_{i}-\psi_{i}, \psi_{j}\right\rangle=\left\langle U \phi_{i}, \psi_{j}\right\rangle-\left\langle\psi_{i}, \psi_{j}\right\rangle=\left\langle U \phi_{i}, U \phi_{j}\right\rangle-\left\langle\psi_{i}, \psi_{j}\right\rangle=\left\langle\phi_{i}, \phi_{j}\right\rangle-\left\langle\psi_{i}, \psi_{j}\right\rangle=0,
$$

which implies $U \phi_{i}=\psi_{i}$ since $\left\{\psi_{1}, \ldots, \psi_{d}\right\}$ is a basis for $\mathcal{H}$.

Theorem 2.9 (Equivalent tight frames). Tight frames $\Phi$ and $\Psi$ in standard form are equivalent if and only if there is a bijection $\pi: \Phi \rightarrow \Psi$ with

$$
\left\langle\phi_{1}, \phi_{2}\right\rangle=\left\langle\pi \phi_{1}, \pi \phi_{2}\right\rangle, \quad \forall \phi_{1}, \phi_{2} \in \Phi
$$

In particular, isometric tight frames are equivalent if and only if after a suitable reordering their Gram matrices are equal.

Proof: $\quad$ Suppose the tight frames are equivalent via some $U$ as in (2.5). Let $\pi \phi:=$ $U \phi$, then

$$
\left\langle\phi_{1}, \phi_{2}\right\rangle=\left\langle U \phi_{1}, U \phi_{2}\right\rangle=\left\langle\pi \phi_{1}, \pi \phi_{2}\right\rangle
$$

Conversely, suppose that (2.10) holds. Then by Lemma 2.7, there is a unitary $U$ with $U \phi=\pi \phi, \forall \phi \in \Phi$, which satisfies (2.5).

Example 4. Let $\omega$ be a primitive third root of unity. Then

$$
\Phi:=\left\{\left[\begin{array}{l}
1 \\
1
\end{array}\right],\left[\begin{array}{c}
\omega \\
\omega^{2}
\end{array}\right],\left[\begin{array}{c}
\omega^{2} \\
\omega
\end{array}\right]\right\}, \quad \Psi:=\left\{\left[\begin{array}{l}
1 \\
1
\end{array}\right],\left[\begin{array}{c}
1 \\
\omega
\end{array}\right],\left[\begin{array}{c}
1 \\
\omega^{2}
\end{array}\right]\right\}
$$

are isometric tight frames for $\mathbb{C}^{\mathbb{}}$. These are examples of what will later be called cyclic frames. These frames are not equivalent since the inner product between any two vectors in $\Phi$ is real, wheras there are two vectors in $\Psi$ with non-real inner product.

In [RW02] it is shown that a frame for $\mathbb{R}^{d}$ is also a frame for $\mathbb{C}^{d}$. Thus the unitary image of the three vectors of (1.1) gives an equivalent tight frame of three vectors for $\mathbb{C}^{\mathbb{Q}}$, which may have complex entries. Here we show how to recognise that such a frame is obtained from a frame for a real space.

Definition. A tight frame $\Phi$ is said to be real if either $\mathcal{H}$ is a real inner product space, or there is a unitary map $U: \mathcal{H} \rightarrow \mathbb{C}^{d}$ with $U \Phi \subset \mathbb{R}^{d}$.

Thus a tight frame is real if it is equivalent to a tight frame for a real inner product space. 
Lemma 2.12. Let $\Phi \subset \mathcal{H}$. There is unitary map $U: \mathcal{H} \rightarrow \mathbb{C}^{d}$ with $U \Phi \subset \mathbb{R}^{d}$ if and only if $\langle\phi, \psi\rangle \in \mathbb{R}, \forall \phi, \psi \in \Phi$.

Proof: $\quad$ If $U \Phi \subset \mathbb{R}^{d}$, then clearly $\langle\phi, \psi\rangle=\langle U \phi, U \psi\rangle \in \mathbb{R}, \forall \phi, \psi \in \Phi$.

Now suppose $\langle\phi, \psi\rangle \in \mathbb{R}, \forall \phi, \psi \in \Phi$. Let $\left\{\phi_{1}, \ldots, \phi_{k}\right\}$ be a basis for $V:=\operatorname{span} \Phi$, and apply the Gram-Schmidt algorithm to obtain an orthonormal basis $\left\{u_{1}, \ldots, u_{k}\right\}$ with $u_{j} \in$ $\operatorname{span}_{\mathbb{R}}\left\{\phi_{1}, \ldots, \phi_{k}\right\}$. This satisfies $\left\langle\phi, u_{j}\right\rangle \in \mathbb{R}, \forall \phi \in \Phi$. Now let $U$ be any unitary matrix mapping the orthonormal vectors $\left\{u_{1}, \ldots, u_{k}\right\}$ to orthonormal vectors $\left\{e_{1}, \ldots, e_{k}\right\} \subset \mathbb{R}^{d}$. Then

$$
U \phi=\sum_{j=1}^{k}\left\langle\phi, u_{j}\right\rangle e_{j} \in \mathbb{R}^{d}, \quad \forall \phi \in \Phi
$$

as required.

Theorem 2.13 (Real frames). A tight frame $\Phi$ is real if and only if

$$
\left\langle\phi_{1}, \phi_{2}\right\rangle \in \mathbb{R}, \quad \forall \phi_{1}, \phi_{2} \in \Phi
$$

Proof: $\quad$ The forward implication is immediate. For the converse, use Lemma 2.12 to choose $U$.

Example 5. Let $\omega$ be a primitive third root of unity. For the isometric tight frames of (2.11) the Gram matrices are given by

$$
\operatorname{Gram}(\Phi)=\left(\begin{array}{ccc}
2 & -1 & -1 \\
-1 & 2 & -1 \\
-1 & -1 & 2
\end{array}\right), \quad \operatorname{Gram}(\Psi)=\left(\begin{array}{ccc}
2 & 1+\omega^{2} & 1+\omega \\
1+\omega & 2 & 1+\omega^{2} \\
1+\omega^{2} & 1+\omega & 2
\end{array}\right)
$$

Thus, by Theorem $2.13, \Phi$ is a real frame (it is a unitary image of three equally spaced vectors in $\mathbb{R}^{2}$ ) and $\Psi$ is not.

\section{Symmetries of tight frames}

Here we introduce the key idea of this paper the symmetry group of a tight frame $\Phi$. This will be the group of invertible linear maps $U: \mathcal{H} \rightarrow \mathcal{H}$ which permute the vectors in $\Phi$. Such a transformation preserves the vector's length, indeed is unitary (orthogonal) since

$$
\left\|U^{*} f\right\|^{2}=\sum_{\phi \in \Phi} c\left|\left\langle U^{*} f, \phi\right\rangle\right|^{2}=\sum_{\phi \in \Phi} c|\langle f, U \phi\rangle|^{2}=\sum_{\phi \in \Phi} c|\langle f, \phi\rangle|^{2}=\|f\|^{2}, \quad \forall f \in \mathcal{H} .
$$

We denote the group of unitary (inner-product preserving) transformations on the real or complex Hilbert space $\mathcal{H}$ by $\mathcal{U}(\mathcal{H})$. The $G$-orbit of a vector $\phi$ under the action of a group $G$ is the set $\{g \phi: g \in G\}$. 
Definition. The symmetry group of a tight frame $\Phi$ is the group

$$
\operatorname{Sym}(\Phi):=\{U \in \mathcal{U}(\mathcal{H}): U \Phi=\Phi\} .
$$

Note that $\operatorname{Sym}(\Phi)$ can only act transitively when the tight frame $\Phi$ is isometric. In this case, we make the following definition:

Definition. An isometric tight frame $\Phi$ is said to be transitive if $G=\operatorname{Sym}(\Phi)$ acts transitively on the set of vectors in $\Phi$, i.e.,

$$
G \phi=\Phi, \quad \forall \phi \in \Phi .
$$

Bölcskei and Eldar [BE03] use the term geometrically uniform for a transitive tight frame defined by $\Phi:=G \phi$ where $G$ is abelian. A set of vectors in $\mathcal{H}$ (not necessarily forming an isometric tight frame) on which some (finite) subgroup of the unitary group acts transitively is called a "group code" by Slepian [S168] (when $\mathcal{H}=\mathbb{R}^{d}$ ) and a "geometrically uniform code" by Forney [For91]. Not all geometrically uniform codes are tight frames

Note that $\operatorname{Sym}(\Phi)$ is isomorphic to a subgroup of the symmetric group on $\Phi$, and so its order divides $|\Phi|$ !, for short

$$
|\operatorname{Sym}(\Phi)||| \Phi \mid ! .
$$

Also, note that since a $U \in \operatorname{Sym}(\Phi)$ is uniquely determined by is action on a basis taken from $\Phi$, we have

$$
|\operatorname{Sym}(\Phi)| \leq|\Phi|(|\Phi|-1) \cdots(|\Phi|-k+1), \quad k:=\operatorname{dim}(\mathcal{H}) .
$$

Example 1. Let $\Phi_{1}=\left(v_{1}, v_{2}, v_{3}\right)$ be the isometric tight frame obtained by taking three equally spaced unit vectors in $\mathbb{R}^{2}$ (with $c=2 / 3$ ), and $\Phi_{2}=\left(-v_{1}, v_{2}, v_{3}\right)$ the isometric tight frame obtained by replacing one vector by its negative.
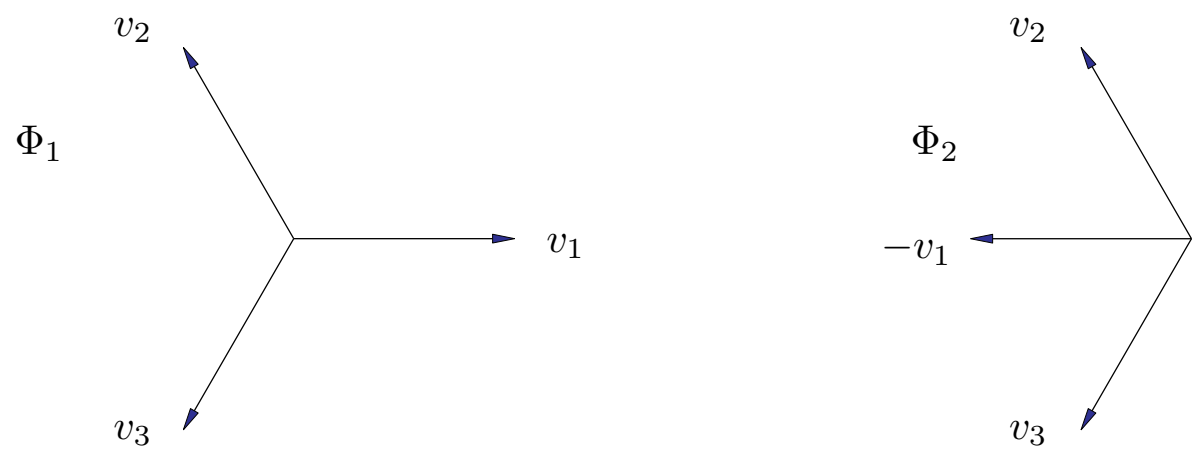

These have symmetry groups $\operatorname{Sym}\left(\Phi_{1}, c\right)=D_{3}$ (the dihedralgroup of order 6) which acts transitively, so $\Phi_{1}$ is a transitive frame, and $\operatorname{Sym}\left(\Phi_{2}, c\right)=C_{2}$ (the cyclic group of order 2).

Example 2. Equivalent tight frames have isomorphic symmetry groups, namely if $\Phi$ is a tight frame and $U \Phi$ is an equivalent tight frame obtained from some unitary $U$, then

$$
\operatorname{Sym}(U \Phi)=U \operatorname{Sym}(\Phi) U^{*} .
$$


Example 3. The isometric tight frames $\Phi$ and $\Psi$ of (2.11) have symmetry groups

$$
\operatorname{Sym}(\Phi)=\left\langle\left(\begin{array}{ll}
0 & 1 \\
1 & 0
\end{array}\right),\left(\begin{array}{cc}
\omega & \\
& \omega^{2}
\end{array}\right)\right\rangle \approx D_{3}, \quad \operatorname{Sym}(\Psi)=\left\langle\left(\begin{array}{ll}
1 & \\
& \omega
\end{array}\right)\right\rangle \approx C_{3} .
$$

In view of $(3.3)$ and the fact $\left|D_{3}\right|=3$ !, no tight frame of 3 vectors in $\mathbb{C}^{\mathbb{E}}$ can have more symmetries than $\Phi$.

Example 4. The symmetry group of the isometric tight frame given by the $n^{\text {th }}$ roots of unity in $\mathbb{R}^{2}$ is isomorphic to the dihedral group

$$
D_{n}:=\left\langle a, b: a^{n}=1, b^{2}=1, b^{-1} a b=a^{n-1}\right\rangle
$$

of order $2 n$, and is generated by a rotation $a$ through $2 \pi / n$ and a reflection $b$.

\section{The Gram matrix of a transitive tight frame}

Recall that an isometric tight frame $\Phi$ is transitive if its symmetry group $\operatorname{Sym}(\Phi)$ acts transitively on $\Phi$, and a tight frame is determined by its Gramian (Theorem 2.7). Thus transitivity should be expressible in terms of the Gram matrix. This is the content of Theorem 4.2 below, which enables us to recognise whether an isometric tight frame is transitive by looking at the entries of its Gramian.

To simplify the presentation, it is convenient (for this section only) to consider tight frames where the vectors may be repeated, i.e., $\Phi$ is a multiset, rather than a set. We will refer to such tight frames as ones with multiplicities.

Definition. We say that $\Phi$ an isometric tight frame with multiplicities is generated by a group $G$ of unitary transformations if

$$
G \cdot \phi:=(g \phi: g \in G)=\Phi \quad \text { (equality of multisets), } \quad \forall \phi \in \Phi .
$$

Clearly, $\Psi$ the set of vectors in an isometric tight frame $\Phi$ with multiplicities generated by $G$ is a transitive frame whose symmetries contain $G$, and for any subgroup $G$ of $\operatorname{Sym}(\Psi)$ which acts transitively on $\Psi$ the multiset $G \cdot \phi, \phi \in \Phi$ is $\Psi$ repeated some number of times.

Definition. Let $G$ be a finite group. We say that a matrix $A=\left[a_{i j}\right]_{i, j=1}^{n}$ is $G$-circulant if there exists a bijection $\{1, \ldots, n\} \rightarrow G: i \mapsto g_{i}$ and a map $\nu: G \rightarrow \mathbb{C}$ for which

$$
a_{i j}=\nu\left(g_{i}^{-1} g_{j}\right), \quad \forall i, j
$$

It is elementary if $\nu$ can not be factored $\nu=\eta \circ \theta$ where $\theta: G \rightarrow H$ is a group homomorphism with $|H|<|G|$ and $\eta: H \rightarrow \mathbb{C}$

When $G$ is a cyclic group a $G$-circulant matrix is a circulant matrix (cf Davis [Da79]). Each row of a $G$-circulant matrix $A$ is a permutation of the values $\nu\left(g_{1}\right), \ldots, \nu\left(g_{n}\right)$, and $\nu$ is constant on the cosets given by the normal subgroup

$$
N:=\left\{g \in G: \nu\left(h_{1} g h_{2}\right)=\nu\left(h_{1} h_{2}\right), \forall h_{1}, h_{2} \in G\right\} .
$$


It can be shown that $A$ is elementary if and only if $N=\{1\}$.

The Gramian of $\Phi$ is a $G$-circulant matrix if and only if there exists a bijection $G \rightarrow \Phi: g \mapsto \phi_{g}$ for which

$$
\left\langle\phi_{h g_{1}}, \phi_{h g_{2}}\right\rangle=\left\langle\phi_{g_{1}}, \phi_{g_{2}}\right\rangle, \quad \forall g_{1}, g_{2}, h \in G .
$$

This can be interpreted as condition of 'equal spacing'.

Theorem 4.2 (Transitive frames). Let $\Phi$ be an isometric tight frame with multiplicities for $\mathcal{H}$. Then the following are equivalent

1. $\Phi$ generated by some group $G \subset \mathcal{U}(\mathcal{H})$.

2. The Gramian of $\Phi$ is an elementary $G$-circulant matrix for some group $G$.

3. There exists a group $G$ and a bijection $G \rightarrow \Phi: g \mapsto \phi_{g}$ for which

$$
\begin{gathered}
\left\langle\phi_{h g_{1}}, \phi_{h g_{2}}\right\rangle=\left\langle\phi_{g_{1}}, \phi_{g_{2}}\right\rangle, \quad \forall g_{1}, g_{2}, h \in G, \\
\phi_{g h}=\phi_{h}, \quad \forall h \in G \quad \Longrightarrow \quad g=1 .
\end{gathered}
$$

The groups $G$ above can be taken to be the same.

Proof: $\quad(1 \Longrightarrow 2)$. Suppose that $\Phi$ is generated by $G \subset \mathcal{U}(\mathcal{H})$, i.e., for each $\phi \in \Phi$,

$$
\Phi=\left(\phi_{1}, \ldots, \phi_{n}\right)=\left(g_{1} \phi, \ldots, g_{n} \phi\right)
$$

where $\{1, \ldots, n\} \rightarrow G: i \mapsto g_{i}$ is a bijection. Then the entries of the Gramian of $\Phi$ are

$$
\left\langle\phi_{i}, \phi_{j}\right\rangle=\left\langle g_{i} \phi, g_{j} \phi\right\rangle=\left\langle\phi, g_{i}^{-1} g_{j} \phi\right\rangle=\nu\left(g_{i}^{-1} g_{j}\right), \quad \nu(g):=\langle\phi, g \phi\rangle, \quad \forall g \in G,
$$

and so it is $G$-circulant. Moreover, it is elementary since

$$
\begin{aligned}
N: & =\left\{g \in G: \nu\left(h_{1} g h_{2}\right)=\nu\left(h_{1} h_{2}\right), \forall h_{1}, h_{2} \in G\right\} \\
& =\left\{g \in G:\left\langle\phi, h_{1} g h_{2} \phi\right\rangle=\left\langle\phi, h_{1} h_{2} \phi\right\rangle, \forall h_{1}, h_{2} \in G\right\} \\
& =\left\{g \in G:\left\langle h_{1}^{-1} \phi, g h_{2} \phi\right\rangle=\left\langle h_{1}^{-1} \phi, h_{2} \phi\right\rangle, \forall h_{1}, h_{2} \in G\right\} \\
& =\left\{g \in G: g h_{2} \phi=h_{2} \phi, \forall h_{2} \in G\right\} \\
& =\{1\} .
\end{aligned}
$$

$(2 \Longrightarrow 3)$. Suppose the Gramian of $\Phi$ is a $G$-circulant matrix, i.e., $\left\langle\phi_{i}, \phi_{j}\right\rangle=\nu\left(g_{i}^{-1} g_{j}\right)$, which is elementary. Let $g \mapsto \phi_{g}$ be the bijection given by $g_{i} \mapsto \phi_{i}$, so $\left\langle\phi_{g_{i}}, \phi_{g_{j}}\right\rangle=\nu\left(g_{i}^{-1} g_{j}\right)$, and we have

$$
\left\langle\phi_{h g_{1}}, \phi_{h g_{2}}\right\rangle=\nu\left(\left(h g_{1}\right)^{-1}\left(h g_{2}\right)\right)=\nu\left(g_{1}^{-1} g_{2}\right)=\left\langle\phi_{g_{1}}, \phi_{g_{2}}\right\rangle, \quad \forall g_{1}, g_{2}, h \in G,
$$

and, since the Gramian is elementary,

$$
\begin{aligned}
N: & =\left\{g \in G: \nu\left(h_{1} g h_{2}\right)=\nu\left(h_{1} h_{2}\right), \forall h_{1}, h_{2} \in G\right\} \\
& =\left\{g \in G:\left\langle\phi_{h_{1}^{-1}}, \phi_{g h_{2}}\right\rangle=\left\langle\phi_{h_{1}^{-1}}, \phi_{h_{2}}\right\rangle, \forall h_{1}, h_{2} \in G\right\} \\
& =\left\{g \in G: \phi_{g h_{2}}=\phi_{h_{2}}, \forall h_{2} \in G\right\} \\
& =\{1\},
\end{aligned}
$$


which implies (4.4).

$(3 \Longrightarrow 1)$. Suppose that such a bijection exists. Let $c>0$ be the common scaling factor for $\Phi$, so that

$$
f=c \sum_{g \in G}\left\langle f, \phi_{g}\right\rangle \phi_{g}, \quad \forall f \in \mathcal{H} .
$$

For each $h \in G$, define a linear operator $U_{h}$ by

$$
U_{h} f:=c \sum_{g \in G}\left\langle f, \phi_{g}\right\rangle \phi_{h g}, \quad \forall f \in \mathcal{H} .
$$

It follows that $U_{h}$ is unitary and satisfies

$$
U_{h} \phi_{g}=\phi_{h g}, \quad \forall g \in G
$$

by the calculations

$$
\begin{aligned}
\left\|U_{h} f\right\|^{2} & =\left\langle c \sum_{g_{1} \in G}\left\langle f, \phi_{g_{1}}\right\rangle \phi_{h g_{1}}, c \sum_{g_{2} \in G}\left\langle f, \phi_{g_{2}}\right\rangle \phi_{h g_{2}}\right\rangle \\
& =c^{2} \sum_{g_{1} \in G} \sum_{g_{2} \in G}\left\langle f, \phi_{g_{1}}\right\rangle \overline{\left\langle f, \phi_{g_{2}}\right\rangle}\left\langle\phi_{h g_{1}}, \phi_{h g_{2}}\right\rangle \\
& =c^{2} \sum_{g_{1} \in G} \sum_{g_{2} \in G}\left\langle f, \phi_{g_{1}}\right\rangle \overline{\left\langle f, \phi_{g_{2}}\right\rangle}\left\langle\phi_{g_{1}}, \phi_{g_{2}}\right\rangle \\
& =\left\langle c \sum_{g_{1} \in G}\left\langle f, \phi_{g_{1}}\right\rangle \phi_{g_{1}}, c \sum_{g_{2} \in G}\left\langle f, \phi_{g_{2}}\right\rangle \phi_{g_{2}}\right\rangle=\|f\|^{2},
\end{aligned}
$$

and

$$
U_{h} \phi_{g}=c \sum_{g_{1} \in G}\left\langle\phi_{g}, \phi_{g_{1}}\right\rangle \phi_{h g_{1}}=c \sum_{g_{1} \in G}\left\langle\phi_{h g}, \phi_{h g_{1}}\right\rangle \phi_{h g_{1}}=\phi_{h g} .
$$

which use (4.9) and (4.5). The map $G \rightarrow \mathcal{U}(\mathcal{H}): g \mapsto U_{g}$ is a group homomorphism, since (4.6) gives

$$
U_{g_{1}} U_{g_{2}}=U_{g_{1} g_{2}}, \quad g_{1}, g_{2} \in G .
$$

Moreover, this is an isomorphism, since by (4.4) its kernel is

$$
\left\{g \in G: U_{g}=1\right\}=\left\{g \in G: U_{g} \phi_{h}=\phi_{h}, \forall h \in G\right\}=\{1\} .
$$

Thus, $\left\{U_{g}: g \in G\right\} \subset \mathcal{U}(\mathcal{H})$ is isomorphic to $G$ and generates $\Phi$ since

$$
\left(U_{g} \phi_{h}: g \in G\right)=\left(\phi_{h g}: g \in G\right)=\Phi, \quad \forall \phi_{h} \in \Phi .
$$


Theorem 4.2 can be stated for frames, i.e., without multiplicities. This requires the notion of augmented $G$-circulant matrices.

Definition. Let $G$ be a finite group. We say that a matrix $A=\left[a_{i j}\right]_{i, j=1}^{n}$ is an augmented $G$-circulant matrix if there is a function $\nu: G \rightarrow$ Cand a subgroup $H$ of $G$ such that

$$
\nu\left(h_{1} g h_{2}\right)=\nu(g) \quad \forall h_{1}, h_{2} \in H \quad \forall g \in G,
$$

and there exists a set $\left\{g_{1}, \ldots g_{n}\right\}$ of left coset representatives of $H$ in $G$ with

$$
a_{i j}=\nu\left(g_{i}^{-1} g_{j}\right) \quad 1 \leq i, j \leq n .
$$

If $H$ is trivial, then $A$ is simply a $G$-circulant matrix.

Theorem 4.8 (Transitive tight frames II). Let $\Phi$ be an isometric tight frame for $\mathcal{H}$. Then the following are equivalent

1. $\Phi$ is transitive.

2. The Gramian of $\Phi$ is an augmented $G$-circulant matrix for some group $G$.

3. There exists a group $G$ and a surjection $\rho: G \rightarrow \Phi: g \mapsto \phi_{g}$ for which all the values $\left|\rho^{-1}\left(\phi_{g}\right)\right|$ are equal, and

$$
\left\langle\phi_{h \gamma_{1}}, \phi_{h \gamma_{2}}\right\rangle=\left\langle\phi_{\gamma_{1}}, \phi_{\gamma_{2}}\right\rangle, \quad \forall \gamma_{1}, \gamma_{2}, h \in G
$$

In particular, if $\Phi$ is $G$-circulant then $\Phi$ is transitive.

Example 1. The Gram matrices of (2.14) are clearly circulant, and hence $G$-circulant for $G=C_{3}$ the cyclic group of order 3 .

Example 2. Let $G \subset \mathcal{U}\left(\mathbb{R}^{2}\right)$ be the nonabelian group generated by $a$ the rotation through $2 \pi / 3$ and $b$ the reflection in the $y$-axis, i.e.,

$$
G:=\left\langle a, b: a^{3}=1, b^{2}=1, b^{-1} a b=a^{2}\right\rangle, \quad a:=\frac{1}{2}\left(\begin{array}{cc}
-1 & -\sqrt{3} \\
\sqrt{3} & -1
\end{array}\right), \quad b:=\left(\begin{array}{cc}
-1 & \\
& 1
\end{array}\right),
$$

which is the dihedral group $D_{3}$. It follows from later results on irreducible groups, that

$$
\Phi:=G \phi=\left\{\phi, a \phi, a^{2} \phi, b \phi, a b \phi, a^{2} b \phi\right\}
$$

is an isometric tight frame for all nonzero $\phi \in \mathbb{R}^{2}$. Suppose $\|\phi\|=1$. If $|\Phi|=6$, then its Gram matrix is

$$
\operatorname{Gram}(\Phi)=\left[\begin{array}{cccccc}
1 & -1 / 2 & -1 / 2 & \alpha+\beta & -\alpha & -\beta \\
-1 / 2 & 1 & -1 / 2 & -\beta & \alpha+\beta & -\alpha \\
-1 / 2 & -1 / 2 & 1 & -\alpha & -\beta & \alpha+\beta \\
\alpha+\beta & -\beta & -\alpha & 1 & -1 / 2 & -1 / 2 \\
-\alpha & \alpha+\beta & -\beta & -1 / 2 & 1 & -1 / 2 \\
-\beta & -\alpha & \alpha+\beta & -1 / 2 & -1 / 2 & 1
\end{array}\right]
$$

where $\phi=(x, y)$, and

$$
-\alpha:=\frac{1}{2} x^{2}-\sqrt{3} x y-\frac{1}{2} y^{2}, \quad-\beta:=\frac{1}{2} x^{2}+\sqrt{3} x y-\frac{1}{2} y^{2}, \quad \alpha+\beta=y^{2}-x^{2} .
$$

Here the entries are indexed in the order given by (4.10), and so the $G$-circulant structure of $\operatorname{Gram}(\Phi)$ is apparent. Otherwise, $\Phi$ is the $3^{\text {rd }}$-roots of unity in $\mathbb{R}^{2}$, cf $(2.14)$. 
Corollary 4.11 (Distinct entries of the Gramian). Let $\Phi$ be a real isometric tight frame generated by a group $G$ which has $\kappa(G)$ elements of order less than or equal to two, and let $N(\Phi)$ be the number of distinct entries of the Gramian of $\Phi$. Then

$$
N(\Phi) \leq \frac{|G|+\kappa(G)}{2} .
$$

Proof: Suppose $\Phi=\{g \phi\}_{g \in G}$. The entries of the Gramian of $\Phi$ are the values of $\nu: G \rightarrow \mathbb{R}: g \mapsto\langle g \phi, \phi\rangle$. Let $A:=\left\{g \in G: g^{2}=1\right\}$. For $g \in G \backslash A$, we have $g^{-1} \in G \backslash A$ with $g^{-1} \neq g$ and

$$
\nu\left(g^{-1}\right)=\left\langle g^{-1} \phi, \phi\right\rangle=\langle\phi, g \phi\rangle=\langle g \phi, \phi\rangle=\nu(g),
$$

so that $\nu$ can take on at most

$$
|A|+\frac{|G \backslash A|}{2}=\kappa(G)+\frac{|G|-\kappa(G)}{2}=\frac{|G|+\kappa(G)}{2}
$$

distinct values.

Since $\kappa(G)$ depends only on the isomorphism class of $G$, this result can be used to show that certain isometric tight frames are not generated by particular abstract groups.

Example 3. Let $\Phi$ be the real isometric tight frame (4.10) of the previous example, where $\phi=(x, y)$ satisfies $x y \neq 0$ and $|x / y| \neq \sqrt{3}, 1 / \sqrt{3}$. Then $\Phi$ consists of 6 distinct vectors, and its Gramian has 5 distinct entries. It cannot therefore be generated by an abelian group of order 6 , since $C_{6}$ is the only abelian group of order 6 and it satisfies

$$
\frac{\left|C_{6}\right|+\kappa\left(C_{6}\right)}{2}=\frac{6+2}{2}=4<5 .
$$

This gives a counterexample to a theorem claimed (but not proved) in [E02:Th. 4]. This result states that a set of real vectors $\Phi$ has a permuted Gram matrix (one in which the rows are permutations of the entries of the first row) if and only if the vectors in $\Phi$ are the orbit of an abelian group of unitary matrices. The Gram matrix of the $\Phi$ of this example is $G$-circulant, and so, in particular, is a permuted matrix. But as we have just proved it is not the orbit of any abelian group of unitary matrices.

\section{Cyclic and Harmonic frames}

The character table of the cyclic group $G=C_{3}$ of order 3 is

$$
\left[\begin{array}{ccc}
1 & 1 & 1 \\
1 & \omega & \omega^{2} \\
1 & \omega^{2} & \omega
\end{array}\right], \quad \omega \neq 1, \quad \omega^{3}=1
$$


which has orthogonal rows/columns of equal length. Since the orthogonal projection of an orthonormal basis is a tight frame and $|\omega|=1$, ruling out rows of the above character table gives a submatrix whose columns are an isometric tight frame, e.g., removing rows $1,2,3$ gives

$$
\left\{\left[\begin{array}{l}
1 \\
1
\end{array}\right],\left[\begin{array}{c}
\omega \\
\omega^{2}
\end{array}\right],\left[\begin{array}{c}
\omega^{2} \\
\omega
\end{array}\right]\right\}, \quad\left\{\left[\begin{array}{l}
1 \\
1
\end{array}\right],\left[\begin{array}{c}
1 \\
\omega
\end{array}\right],\left[\begin{array}{c}
1 \\
\omega^{2}
\end{array}\right]\right\}, \quad\left\{\left[\begin{array}{l}
1 \\
1
\end{array}\right],\left[\begin{array}{c}
1 \\
\omega^{2}
\end{array}\right],\left[\begin{array}{c}
1 \\
\omega
\end{array}\right]\right\}
$$

which are the isometric tight frames for $\mathbb{Q}^{\mathbb{Z}}$ of $(2.11)$.

Here we show the same construction works for any abelian group $G$, and refer to the resulting isometric tight frames as 'harmonic frames' (and 'cyclic frames' when $G$ is cyclic). We will show that the harmonic frames are precisely those isometric tight frames which are orbits of an abelian group (of symmetries), and that real cyclic frames of $n$ vectors in $\mathbb{R}^{d}$ exist for all $n \geq d$.

The following basic facts can be found in any standard book on character theory or Fourier analysis on abelian groups, e.g., [JL93] and [Ru62]. Let $G$ be a finite abelian group. The characters of $G$ are the group homomorphisms $\xi: G \rightarrow \mathbb{C} \backslash\{0\}$, where $\mathbb{C} \backslash\{0\}$ is a group under multiplication. Here we think of them as vectors $\xi \in \mathbb{C}^{\mathbb{G}}$ (with the Euclidean inner product), which satisfy

$$
\xi\left(g_{1}+g_{2}\right)=\xi\left(g_{1}\right) \xi\left(g_{2}\right), \quad \forall g_{1}, g_{2} \in G
$$

It follows from (5.1) that $\xi(g)$ is a $|G|$-th root of unity. It can be shown that the characters of $G$ form an orthogonal basis for $\mathbb{C}$, and the square matrix with these vectors as rows is referred to as the character table of $G$ (and by some as the Fourier matrix when $G$ is cyclic).

Definition. Let $G$ be a finite abelian group with characters $\left(\xi_{j}\right)_{j=1}^{n}, J \subset\{1, \ldots, n\}$ and $U: \mathbb{C}^{J} \rightarrow \mathcal{H}$ unitary. Then the isometric tight frame for $\mathcal{H}$ given by

$$
\Phi:=U\left\{\left(\xi_{j}(g)\right)_{j \in J}: g \in G\right\}
$$

is called a harmonic frame. If $G$ is cyclic, then $\Phi$ is called a cyclic frame.

This definition extends those of [CK01]. There $G$ is the cyclic group of order $n$ and the $\Phi$ of (5.2) is called a harmonic tight frame when $U=I$ and a general harmonic frame when $U=D$ with $D$ diagonal (these are both cyclic frames in our terminology).

Example 1. The character table of the cyclic group of order $n$ is

$$
\left[\begin{array}{ccccc}
1 & 1 & 1 & \cdots & 1 \\
1 & \omega & \omega^{2} & \cdots & \omega^{n-1} \\
1 & \omega^{2} & \omega^{4} & \cdots & \omega^{2(n-1)} \\
\vdots & \vdots & \vdots & & \vdots \\
1 & \omega^{n-1} & \omega^{2(n-1)} & \cdots & \omega^{(n-1)(n-1)}
\end{array}\right]
$$


where $\omega$ is primitive $n$-th root of unity. Since $\omega^{-j}=\bar{\omega}^{j}$, taking the second and last rows of the table gives the harmonic frame for $\mathbb{C}^{\mathbb{E}}$

$$
\left\{\left[\begin{array}{l}
1 \\
1
\end{array}\right],\left[\begin{array}{c}
\omega \\
\bar{\omega}
\end{array}\right],\left[\begin{array}{c}
\omega^{2} \\
\bar{\omega}^{2}
\end{array}\right], \ldots,\left[\begin{array}{c}
\omega^{n-1} \\
\bar{\omega}^{n-1}
\end{array}\right]\right\}
$$

which was given in [B98:Sect. 4, Ex. 1]. This is a real cyclic frame, since

$$
\left\langle\left[\begin{array}{c}
\omega^{j} \\
\bar{\omega}^{j}
\end{array}\right],\left[\begin{array}{c}
\omega^{k} \\
\bar{\omega}^{k}
\end{array}\right]\right\rangle=\omega^{j} \bar{\omega}^{k}+\bar{\omega}^{j} \omega^{k}=2 \Re\left(\omega^{j-k}\right) \in \mathbb{R}
$$

which is equivalent to the $n^{\text {th }}$ roots of unity in $\mathbb{R}^{2}$.

Example 2. The smallest noncyclic abelian group is $C_{2} \times C_{2}$. Its character table is

$$
\left[\begin{array}{cccc}
1 & 1 & 1 & 1 \\
1 & -1 & 1 & -1 \\
1 & 1 & -1 & -1 \\
1 & -1 & -1 & 1
\end{array}\right]
$$

Taking any pair of the last three rows gives the harmonic frame

$$
\left\{\left[\begin{array}{l}
1 \\
1
\end{array}\right],\left[\begin{array}{c}
-1 \\
1
\end{array}\right],\left[\begin{array}{c}
-1 \\
-1
\end{array}\right],\left[\begin{array}{c}
1 \\
-1
\end{array}\right]\right\}
$$

of four vectors in $\mathbb{R}^{2}$, and taking the first and any other gives

$$
\left\{\left[\begin{array}{l}
1 \\
1
\end{array}\right],\left[\begin{array}{c}
1 \\
-1
\end{array}\right]\right\}
$$

which is an orthogonal basis.

Example 3. There exist harmonic frames which are not cyclic. Let $\omega$ be a primitive third root of unity, then the following 2 rows of the character table for $C_{3} \times C_{3}$ give a harmonic frame for $\mathbb{C}^{\mathbb{Q}}$ which is not cyclic

$$
\Phi=\left\{\left[\begin{array}{l}
1 \\
1
\end{array}\right],\left[\begin{array}{c}
\omega \\
1
\end{array}\right],\left[\begin{array}{c}
\omega^{2} \\
1
\end{array}\right],\left[\begin{array}{c}
1 \\
\omega
\end{array}\right],\left[\begin{array}{c}
1 \\
\omega^{2}
\end{array}\right],\left[\begin{array}{c}
\omega \\
\omega
\end{array}\right],\left[\begin{array}{c}
\omega \\
\omega^{2}
\end{array}\right],\left[\begin{array}{c}
\omega^{2} \\
\omega
\end{array}\right],\left[\begin{array}{c}
\omega^{2} \\
\omega^{2}
\end{array}\right]\right\}
$$

This can be proved by showing any unitary matrix which permutes these vectors must have order dividing 6. (Briefly, let

$$
g=\left(\begin{array}{ll}
a & b \\
c & d
\end{array}\right) \in \operatorname{Sym}(\Phi)
$$

Then $\left\{a+b, a+b \omega, a+b \omega^{2}\right\} \subset\left\{1, \omega, \omega^{2}\right\}$, whence $a=0$ or $b=0$. Similarly, $c=0$ or $d=0$ and the nonzero entries of $g$ are 3 rd roots of unity). 
Definition. We say that $\Phi$ an isometric tight frame is generated by a group $G$ of unitary transformations if $\Phi$ is a $G$-orbit, i.e.,

$$
G \phi:=\{g \phi: g \in G\}=\Phi, \quad \forall \phi \in \Phi .
$$

If $\Phi$ is generated by an abelian group $G$, then $|\Phi|=|G|$ since for $g_{1}, g_{2} \in G$ :

$$
g_{1} \phi=g_{2} \phi \quad \Longrightarrow \quad g_{1} h \phi=g_{2} h \phi, \quad \forall h \in G \quad \Longrightarrow \quad g_{1}=g_{2} .
$$

Theorem 5.4 (Harmonic frames). Let $\Phi$ be an isometric tight frame for $\mathcal{H}$. Then the following are equivalent

1. $\Phi$ is harmonic (obtained from the character table of $G$ ) and $|\Phi|=|G|$.

2. $\Phi$ is generated by an abelian group $G \subset \mathcal{U}(\mathcal{H})$.

3. There exists an abelian group $G$ and a bijection $G \rightarrow \Phi, g \mapsto \phi_{g}$ for which

$$
\left\langle\phi_{h g_{1}}, \phi_{h g_{2}}\right\rangle=\left\langle\phi_{g_{1}}, \phi_{g_{2}}\right\rangle, \quad \forall g_{1}, g_{2}, h \in G \text {. }
$$

The group $G$ in each equivalence can be taken to be the same, but it need not be unique.

Proof: $\quad(1 \Longrightarrow 2)$. Suppose without loss of generality that the $\Phi$ of $(5.2)$ is

$$
\Phi=\left\{\left(\xi_{j}(g)\right)_{j \in J}: g \in G\right\} .
$$

For each $g \in G$, define a diagonal unitary $J \times J$ matrix by $U_{g}:=\operatorname{diag}\left(\xi_{j}(g): j \in J\right)$. Since $\xi_{j}\left(g_{1} g_{2}\right)=\xi_{j}\left(g_{1}\right) \xi_{j}\left(g_{2}\right)$, the map $g \mapsto U_{g}$ is a group homomorphism, and the group $U_{G}:=\left\{U_{g}: g \in G\right\}$ generates $\Phi$, since

$$
U_{g}\left(\left(\xi_{j}(h)\right)_{j \in J}\right)=\left(\xi_{j}(g) \xi_{j}(h)\right)_{j \in J}=\left(\xi_{j}(g h)\right)_{j \in J}, \quad \forall g, h \in G .
$$

Since the vectors in $\Phi$ are distinct, only $U_{1}$ leaves them unchanged, so the kernel of $g \mapsto U_{g}$ is $\{1\}$ and $U_{G}$ is an isomorphic image of $G$.

$(2 \Longrightarrow 1)$. Suppose that $\Phi$ is generated by an abelian group $G \subset \mathcal{U}(\mathcal{H})$. For such a commuting family of normal matrices there is a unitary $V$ for which all $D_{g}:=V g V^{*}$ are diagonal (with diagonal entries of modulus 1), see, e.g., [HJ85:Th. 2.5.5]. Hence, for $\phi \in \Phi$,

$$
\Phi=\{g \phi: g \in G\}=V^{*} \Psi, \quad \Psi:=\left\{D_{g} \psi: g \in G\right\}, \quad \psi:=V \phi .
$$

Since $\Psi$ is a unitary image of $\Phi, \Psi$ is an isometric tight frame, and so the matrix with these vectors as columns has orthogonal rows of equal length. The length of row $j$ is $\sqrt{n}\left|\psi_{j}\right|>0$, so the components of $\psi$ have equal modulus, and we may write

$$
\Phi=V^{*} \operatorname{diag}\left(\psi_{1}, \ldots, \psi_{d}\right)\left\{D_{g} \overrightarrow{1}: g \in G\right\}, \quad \overrightarrow{1}:=(1, \ldots, 1)^{T},
$$

where $\operatorname{diag}\left(\psi_{1}, \ldots, \psi_{d}\right)$ is a scalar multiple of a unitary matrix. It remains only to show

$$
\xi_{j}: g \mapsto\left(D_{g} \overrightarrow{1}\right)_{j}=\left(D_{g}\right)_{j j}, \quad 1 \leq j \leq d
$$


are characters of $G$, which follows since

$$
\xi_{j}\left(g_{1} g_{2}\right)=\left(D_{g_{1} g_{2}}\right)_{j j}=\left(D_{g_{1}} D_{g_{2}}\right)_{j j}=\left(D_{g_{1}}\right)_{j j}\left(D_{g_{2}}\right)_{j j}=\xi_{j}\left(g_{1}\right) \xi_{j}\left(g_{2}\right)
$$

$(2 \Longrightarrow 3)$. Suppose $\Phi$ is generated by $G$. Define $\phi_{g}:=g \phi$. This is a bijection $G \rightarrow \Phi$ that satisfies the required condition.

$(3 \Longrightarrow 2)$. Suppose $(3)$ holds. Then by the proof of $(4.2), \Phi$ is generated by a group $U_{G}$ which is a homomorphic image of $G$, hence is abelian. In the notation of Theorem 4.2, $U_{G}=\left\{U_{g}: g \in G\right\}$ where $U_{g} \phi_{h}=\phi_{g h}$ for $g, h \in G$. Since $g \mapsto \phi_{g}$ is a bijection, we see that $g \mapsto U_{g}$ is in fact an isomorphism $G \rightarrow U_{G}$.

Example 4. To show that the group $G$ in Theorem 5.4 need not be unique, let $\Phi$ be the isometric tight frame of 4 equally spaced vectors in $\mathbb{R}^{2}$

$$
\Phi:=\left\{\left[\begin{array}{l}
1 \\
0
\end{array}\right],\left[\begin{array}{l}
0 \\
1
\end{array}\right],\left[\begin{array}{c}
-1 \\
0
\end{array}\right],\left[\begin{array}{c}
0 \\
-1
\end{array}\right]\right\}
$$

which has symmetry group $\operatorname{Sym}(\Phi)=D_{4} \subset \mathcal{U}\left(\mathbb{R}^{2}\right)$ as in $(3.5)$, where

$$
a=\left(\begin{array}{cc}
0 & -1 \\
1 & 0
\end{array}\right), \quad b=\left(\begin{array}{ll}
0 & 1 \\
1 & 0
\end{array}\right)
$$

It is generated by the nonisomorphic abelian subgroups

$$
\left\langle a^{2}, b\right\rangle \approx C_{2} \times C_{2}, \quad\langle a\rangle \approx C_{4}
$$

Corollary 5.5 (Cyclic frames). Let $\Phi$ be an isometric tight frame of $n$ vectors in $\mathbb{C}^{d}$ Then the following are equivalent

1. $\Phi$ is cyclic (obtained from the character table of a cyclic group $G$ with $|G|=|\Phi|$ ).

2. $\Phi$ is generated by a cyclic group.

3. There is a bijection $\mathbb{Z}_{n} \rightarrow \Phi, j \mapsto \phi_{j}$ for which

$$
\left\langle\phi_{j}, \phi_{k}\right\rangle=\left\langle\phi_{j+l}, \phi_{k+l}\right\rangle, \quad \forall j, k, l \in \mathbb{Z}_{n}
$$

4. $\Phi$ can be ordered $\left(\phi_{1}, \ldots, \phi_{n}\right)$ so that

$$
\left\langle\phi_{j}, \phi_{k}\right\rangle=\left\langle\phi_{j+1}, \phi_{k+1}\right\rangle, \quad \forall 1 \leq j, k<n
$$

The equivalence of 1 and 4 above was proved in [CK01:Prop. 2.4]. 
Corollary 5.6 (Existence of real cyclic frames). For all $d$ and $n \geq d$, there exists a cyclic frame of $n$ vectors in $\mathbb{R}^{d}$.

Proof: $\quad$ Let $\omega$ be a primitive $n$-th root of unity, and

$$
v:=\left[\begin{array}{c}
\omega \\
\bar{\omega} \\
\omega^{2} \\
\bar{\omega}^{2} \\
\vdots \\
\omega^{d / 2} \\
\bar{\omega}^{d / 2}
\end{array}\right] \quad(d \text { even }) \quad v:=\left[\begin{array}{c}
1 \\
\omega \\
\bar{\omega} \\
\omega^{2} \\
\bar{\omega}^{2} \\
\vdots \\
\omega^{(d-1) / 2} \\
\bar{\omega}^{(d-1) / 2}
\end{array}\right] \quad(d \text { odd })
$$

Then $\Phi:=\left(\phi_{j}\right)_{j=0}^{n-1}, \phi_{j}:=\left(v_{1}^{j}, \ldots, v_{d}^{j}\right)^{T}$ is a cyclic frame for $\mathbb{C}^{\mathbb{d}}$, generated by the cyclic group $G=\langle g\rangle$, where

$$
g:=\left(\begin{array}{llll}
v_{1} & & & \\
& v_{2} & & \\
& & \ddots & \\
& & & v_{d}
\end{array}\right) .
$$

The inner product of any two elements of $\Phi$ is a sum of $n$-th roots of unity and their conjugates, which is real. Hence by Theorem 2.13, there exists a unitary $U$ mapping $\Phi$ into $\mathbb{R}^{d}$, and so giving a real cyclic frame.

This real tight frame has been considered by several authors, including [Z01] and [CFKLT03] (who refer to it as the real harmonic frame).

Example 5. The unitary $U$ of Corollary 5.6 can be constructed taking a block diagonal matrix with all diagonal blocks given by

$$
B:=\frac{1}{\sqrt{2}}\left(\begin{array}{cc}
1 & 1 \\
-i & i
\end{array}\right), \quad B\left[\begin{array}{c}
z \\
\bar{z}
\end{array}\right]=\sqrt{2}\left[\begin{array}{c}
\Re z \\
\Im z
\end{array}\right],
$$

except for $d$ odd, when the last block is [1]. For example, when $d$ is odd and $\omega:=e^{2 \pi i / n}$, multiplying $\left[\phi_{0}, \ldots, \phi_{n-1}\right]$ by $\frac{1}{\sqrt{2}} U$ gives

$$
\left[\begin{array}{ccccc}
1 & \cos \left(2 \pi \frac{1}{n}\right) & \cos \left(2 \pi \frac{2}{n}\right) & \cdots & \cos \left(2 \pi \frac{n-1}{n}\right) \\
0 & \sin \left(2 \pi \frac{1}{n}\right) & \sin \left(2 \pi \frac{2}{n}\right) & \cdots & \sin \left(2 \pi \frac{n-1}{n}\right) \\
1 & \cos \left(2 \pi \frac{2}{n}\right) & \cos \left(2 \pi \frac{4}{n}\right) & \cdots & \cos \left(2 \pi \frac{2(n-1)}{n}\right) \\
0 & \sin \left(2 \pi \frac{2}{n}\right) & \sin \left(2 \pi \frac{4}{n}\right) & \cdots & \sin \left(2 \pi \frac{2(n-1)}{n}\right) \\
\vdots & \vdots & \vdots & & \vdots \\
1 & \cos \left(2 \pi \frac{k}{n}\right) & \cos \left(2 \pi \frac{2 k}{n}\right) & \cdots & \cos \left(2 \pi \frac{(n-1) k}{n}\right) \\
0 & \sin \left(2 \pi \frac{k}{n}\right) & \sin \left(2 \pi \frac{2 k}{n}\right) & \cdots & \sin \left(2 \pi \frac{(n-1) k}{n}\right) \\
\frac{1}{\sqrt{2}} & \frac{1}{\sqrt{2}} & \frac{1}{\sqrt{2}} & \cdots & \frac{1}{\sqrt{2}}
\end{array}\right], \quad k:=\frac{d-1}{2} .
$$


This example is well-known and more details can be found in [GVT98] and [Z01]. We can now add the observation that this isometric tight frame is generated by a cyclic group of order $n$.

Example 6. The $d+1$ vertices of the regular simplex in $\mathbb{R}^{d}$ form a cyclic frame, namely the one obtained by deleting the first row of 1's in (5.3).

It is possible to obtain tight frames from the character table of a nonabelian group in a similar way to our construction of harmonic frames. However, the tight frames so obtained are in general not isometric, and seem not to have symmetries related to the group they were obtained from.

\section{Construction of transitive tight frames}

Here we answer the question: given a finite subgroup $G$ of $\mathcal{U}(\mathcal{H})$, which $G$-orbits are isometric tight frames? Such frames are in general not harmonic. Our results are later used to construct examples of transitive tight frames with given symmetries.

Many highly symmetric configuarations of vectors such as the $n^{\text {th }}$ roots of unity in $\mathbb{R}^{2}$ and the vertices of the platonic solids in $\mathbb{R}^{3}$ are known to be isometric tight frames with large symmetry groups (see eg. [BF03]). We first show how to construct such examples as group orbits.

Definition 6.1. A finite group $G \subset \mathcal{U}(\mathcal{H})$ is irreducible if for every $\phi \neq 0, \phi \in \mathcal{H}$

$$
\operatorname{span}(G \phi)=\mathcal{H}
$$

otherwise it is said to be reducible.

Theorem 6.3 (Irreducible frames). Let $G \subset \mathcal{U}(\mathcal{H})$ be an irreducible finite group. Then the $G$-orbit of every nonzero $\phi \in \mathcal{H}$ is an isometric tight frame for $\mathcal{H}$.

Proof: $\quad$ Suppose $G$ is irreducible. Let $\phi \in \mathcal{H}, \phi \neq 0$, and define a linear map $S_{\phi}: \mathcal{H} \rightarrow \mathcal{H}$ by

$$
S_{\phi}(f):=\sum_{g \in G}\langle f, g \phi\rangle g \phi
$$

As each element of $G \phi$ occurs the same number of times in the list $(g \phi)_{g \in G}$, we need only show that $S_{\phi}$ is a scalar multiple of the identity. It is easily shown that $S_{\phi}$ is positive definite, hence has a positive eigenvalue $\lambda$. It is also easy to see that $S_{\phi}$ commutes with every element of $G$, hence the $\lambda$ - eigenspace $\operatorname{ker}(S-\lambda I)$ is a nontrivial $G$-invariant subspace of $\mathcal{H}$, which must be all of $\mathcal{H}$ since $G$ is irreducible. Thus

$$
f=\frac{1}{\lambda} S_{\phi}(f)=\frac{1}{\lambda} \sum_{g \in G}\langle f, g \phi\rangle g \phi=c \sum_{\psi \in G \phi}\langle f, \psi\rangle \psi, \quad \forall f \in \mathcal{H},
$$

for some $c>0$, so $G \phi$ is an isometric tight frame 
A more detailed proof of this result, together with an historical discussion, can be found in [VW03]. In that paper, we obtain as immediate applications of (6.3) the facts that the $n^{\text {th }}$ roots of unity, vertices of the platonic solids, and vertices of the truncated icosahedron are tight frames. Of course, there are many other examples.

We now show that there are many examples of isometric tight frames generated by irreducible groups which are not harmonic frames, and therefore cannot be generated by an abelian group.

Theorem 6.4. Suppose $G \subset \mathcal{U}(\mathcal{H})$ is an irreducible group, and let $S:=\{\phi:\|\phi\|=1\}$. For each $g \in G$, define a map $f_{g}: S \rightarrow \mathbb{F}$ by

$$
f_{g}(\phi):=\langle\phi, g \phi\rangle, \quad \forall \phi \in S
$$

If $f_{g}$ is not constant for some choice of $g$, then some $G$-orbit is an isometric tight frame for $\mathcal{H}$ which is not generated by an abelian group.

Proof: Suppose some such $f_{g}$ is not constant. Then $f_{g}(S)$ is a connected subset of $\mathbb{F}$ (the continuous image of a connected set) containing two distinct points, and so $f_{g}(S)$ is uncountable. Thus there exists a $\phi \in S$ for which $f_{g}(\phi)=\langle\phi, g \phi\rangle$ is not a sum of roots of unity (as only countably many such sums exist). Therefore $G \phi$ is not a harmonic frame, and so, by Theorem 5.4, is not generated by an abelian group.

Given a group $G \subset \mathcal{U}(\mathcal{H})$, it need not be the case that every $G$-orbit is a tight frame. We now show that if some $G$-orbit spans $\mathcal{H}$, then there is a $G$-orbit which is a tight frame. This result was also given in [BE03], for the case of abelian $G$.

Theorem 6.5. Let $G \subset \mathcal{U}(\mathcal{H})$ be a finite group, $v$ a vector whose $G$-orbit spans $\mathcal{H}$, and define

$$
\phi:=S^{-1 / 2} v, \quad S f:=\sum_{g \in G}\langle f, g v\rangle g v
$$

Then $G \phi$ is a transitive isometric tight frame (whose symmetry group contains $G$ ).

Proof: It is well-known that $\Psi:=\left\{S^{-1 / 2} g v\right\}_{g \in G}$ is a tight frame (it is the "associated tight frame" of the spanning set $G \phi$ ). It is easy to show that $S$ commutes with every $g \in G$, hence so does $S^{-1 / 2}$ (since $S^{-1 / 2}$ can be written as a power series in $S$ ). Therefore, $\Psi=G S^{-1 / 2} v$ is a transitive isometric tight frame generated by $G$, as required.

Thus we now know that given a finite group $G \subset \mathcal{U}(\mathcal{H})$ :

1. Every $G$-orbit spans $\mathcal{H}$ if and only if every $G$-orbit is a tight frame (Theorem 6.3).

2. There exits a $G$-orbit which spans $\mathcal{H}$ if and only if there exists a $G$-orbit which is a tight frame (Theorem 6.5).

For case 2, we now determine exactly which $G$-orbits are tight frames. For this, it is natural to use the language of $\mathbb{F} G$-modules (representation theory). 
Definition. Let $V$ be a vector space over $\mathbb{F}$ and $G$ be a group. Then $V$ is said to be an $\mathbb{F} G$-module if there is multiplication $g v, g \in G, v \in V$ which is an action of $G$ on $V$ with $v \mapsto g v$ being a linear map, i.e., satisfying for all $u, v \in V, \alpha, \beta \in \mathbb{F}$ and $g, h \in G$

1. $g v \in V,(g h) v=g(h v), 1 v=v$.

2. $g(\alpha u+\beta v)=\alpha(g u)+\beta(g v)$.

We are concerned only with the case when $G$ acts on $\mathcal{H}$ as unitary transformations, i.e., each $g \in G$ induces a unitary transformation $\mathcal{H} \rightarrow \mathcal{H}: v \mapsto g v$.

Definition. A subspace $W$ of an $\mathbb{F} G$-module $V$ is called an $\mathbb{F} G$-submodule of $V$ if it is $G$-invariant, i.e., $g w \in W, \forall g \in G, \forall w \in W$.

Definition. An $\mathbb{F} G$-module $V$ is irreducible if $V$ is not zero and has no $\mathbb{F} G$-submodules apart from $\{0\}$ and $V$.

Thus an irreducible $\mathbb{F} G$-submodule is precisely a minimal nontrivial $G$-invariant subspace, i.e., a subspace $W$ of $V$ for which $\operatorname{span}(G w)=W, \forall w \in W \backslash\{0\}$.

Definition. A linear transformation $\sigma: V_{i} \rightarrow V_{j}$ between $\mathbb{F} G$-modules $V_{i}$ and $V_{j}$ is said to be an $\mathbb{F} G$-homomorphism if it satisfies $\sigma g=g \sigma, \forall g \in G$. A bijective $\mathbb{F} G-$ homomorphism is called an $\mathbb{F} G$-isomorphism and we write $V_{i} \approx V_{j}$ to mean that there exists an $\mathbb{F} G$-isomorphism $\sigma: V_{i} \rightarrow V_{j}$.

We now give a version of Maschke's theorem: that an $\mathbb{F} G$-module can be written as an algebraic direct sum of irreducible submodules, where the fact that $G$ acts as unitary transformations allows us to obtain an orthogonal sum.

Lemma 6.6 (Maschke). Let $G$ be a finite group which acts on $V=\mathcal{H}$ as unitary transformations, e.g., $G \subset \mathcal{U}(\mathcal{H})$. Then the $\mathbb{F} G$-module $V$ can be written as an orthogonal direct sum $V=V_{1} \oplus V_{2} \oplus \cdots \oplus V_{k}$ of irreducible $\mathbb{F} G$-submodules $V_{i}$, where the summands are unique up to ordering and $\mathbb{F} G$-isomorphism, and the homogeneous components

$$
H_{V}(W):=\sum_{\substack{X \simeq V \\ X \approx W}} X=\bigoplus_{V_{j} \approx W} V_{j}
$$

corresponding to an irreducible $W$ are unique.

Proof: $\quad$ We use strong induction on $d=\operatorname{dim}(\mathcal{H})$. The result is trivial for $d=0$. Suppose $d>0$ and let $V_{1} \neq\{0\}$ be an irreducible $\mathbb{F} G$-submodule of $V$. Then $V=V_{1} \oplus W$ with $W$ the orthogonal complement of $V_{1}$. We now show $W$ is an $\mathbb{F} G$-submodule of $V$, which will complete the proof.

Let $g \in G, w \in W$, then

$$
\langle g w, v\rangle=\left\langle w, g^{-1} v\right\rangle=0, \quad \forall v \in V_{1}
$$

since $g^{-1} v \in V_{1}$, and so $g w \in V_{1}^{\perp}=W$.

It is easy to show the $V_{j}$ are unique upto $\mathbb{F} G$-isomorphism (Jordan-Hölder), and that $H_{V}(W)=\bigoplus_{V_{j} \approx W} V_{j}$. 
Thus each $V$ has a unique orthogonal decomposition into its homogeneous components

$$
V=\bigoplus_{W \in \mathcal{W}} H_{V}(W)
$$

where $\mathcal{W}$ is the collection of different irreducible $W$ which appear as submodules of $V$.

Lemma 6.7. Let $G$ be a finite group which acts on $\mathcal{H}$ as unitary transformations, and $\mathcal{H}=V_{1} \oplus V_{2} \oplus \cdots \oplus V_{k}$ an orthogonal direct sum of irreducible $\mathbb{F} G$-modules. Then $G v$, $v=v_{1}+v_{2}+\cdots+v_{k}, v_{i} \in V_{i}$, is an isometric tight frame for $\mathcal{H}$ if and only if

$$
\begin{gathered}
\frac{\left\|v_{i}\right\|^{2}}{\left\|v_{j}\right\|^{2}}=\frac{\operatorname{dim}\left(V_{i}\right)}{\operatorname{dim}\left(V_{j}\right)}, \quad \forall 1 \leq i, j \leq k . \\
\sum_{g \in G}\left\langle v_{i}, g v_{i}\right\rangle g v_{j}=0, \quad \forall i \neq j .
\end{gathered}
$$

Proof: $\quad$ Since each element of $G v$ appears the same number of times in the list $(g v)_{g \in G}$, we know that $G v$ is an isometric tight frame for $\mathcal{H}$ if and only if there exists a $\lambda>0$ such that

$$
\sum_{g \in G}\langle f, g v\rangle g v=\lambda f, \quad \forall f \in \mathcal{H}
$$

We need only check this for $f=f_{i} \in V_{i}, 1 \leq i \leq k$. We have

$$
\sum_{g \in G}\left\langle f_{i}, g v\right\rangle g v=\sum_{g \in G}\left\langle f_{i}, g v\right\rangle \sum_{j=1}^{k} g v_{j}=\sum_{g \in G}\left\langle f_{i}, g v_{i}\right\rangle g v_{i}+\sum_{j \neq i}\left\langle f_{i}, g v_{j}\right\rangle g v_{j}
$$

Equating the $V_{j}$ components of (6.10), we see that (6.10) equals $\lambda f_{i}$ if and only if the following hold

$$
\begin{gathered}
\sum_{g \in G}\left\langle f_{i}, g v_{i}\right\rangle g v_{i}=\lambda f_{i}, \\
\sum_{g \in G}\left\langle f_{i}, g v_{j}\right\rangle g v_{j}=0 \quad \forall j \neq i .
\end{gathered}
$$

To check (6.11), observe that for $1 \leq i \leq k$, the restiction $\left.G\right|_{V_{i}}$ of $G$ to $V_{i}$ is a subgroup of $\mathcal{U}\left(V_{i}\right)$ acting irreducibly on $V_{i}$, so we can apply (6.3) to obtain

$$
\sum_{g \in G}\left\langle f_{i}, g v_{i}\right\rangle g v_{i}=\lambda_{i} f_{i}
$$

for some $\lambda_{i}>0$. To calculate $\lambda_{i}$, let $\left\{e_{j}\right\}_{1 \leq j \leq \operatorname{dim}\left(V_{i}\right)}$ be an orthonormal basis of $V_{i}$. Then

$$
\sum_{g \in G}\left\langle e_{j}, g v_{i}\right\rangle g v_{i}=\lambda_{i} e_{j}, \quad 1 \leq j \leq \operatorname{dim}\left(V_{i}\right)
$$


which implies

$$
\sum_{j} \sum_{g \in G}\left|\left\langle e_{j}, g v_{i}\right\rangle\right|^{2}=\lambda_{i} \operatorname{dim}\left(V_{i}\right)=\sum_{g \in G}\left\|g v_{i}\right\|^{2}=|G|\left\|v_{i}\right\|^{2}
$$

Hence

$$
\lambda_{i}=\frac{|G|\left\|v_{i}\right\|^{2}}{\operatorname{dim}\left(V_{i}\right)}
$$

So (6.11) holds if and only if

$$
\lambda=\lambda_{i}=\frac{|G|\left\|v_{i}\right\|^{2}}{\operatorname{dim}\left(V_{i}\right)}, \quad 1 \leq i \leq k \quad \Longleftrightarrow \quad \frac{\left\|v_{i}\right\|^{2}}{\operatorname{dim}\left(V_{i}\right)}=\frac{\left\|v_{j}\right\|^{2}}{\operatorname{dim}\left(V_{j}\right)}, \quad 1 \leq i, j \leq k
$$

as required.

Similarly, (6.12) holds $\forall f_{i} \in V_{i}$ iff it holds for the spanning set $\left\{h v_{i}\right\}_{h \in G} \subset V_{i}$, i.e.

$$
\sum_{g \in G}\left\langle h v_{i}, g v_{i}\right\rangle g v_{j}=h \sum_{g \in G}\left\langle v_{i}, h^{-1} g v_{i}\right\rangle h^{-1} g v_{j}=0,
$$

giving (6.9).

We now describe a special case of the orthogonal decomposition of $\mathcal{H}$ into irreducible $\mathbb{F} G$-modules, which makes (6.9) easy to check.

Let $\operatorname{Hom}_{\mathbb{F} G}(V, W)$ be the space of $\mathbb{F} G$-homomorphisms from $V$ to $W$, which is a finite dimensional vector space over $\mathbb{F}$.

Lemma 6.13 (Schur). If $V$ and $W$ are irreducible $\mathbb{F} G$-modules, with $G$ finite, then $\operatorname{Hom}_{\mathbb{F} G}(V, W)=0$ if $V$ and $W$ are not $\mathbb{F} G$-isomorphic, otherwise $\operatorname{Hom}_{\mathbb{F} G}(V, W)$ is a division ring.

An $\mathbb{R} G$-module can be made into a $\mathbb{C} G$-module by complexifying the underlying vector space in the usual way. Thus an $\mathbb{F} G$-module $V$ can always be thought of as a $\mathbb{C} G$-module. If $V$ is irreducible as a $\mathbb{C} G$-module then it is said to be absolutely irreducible.

Lemma 6.14. Let $G$ be a finite subgroup of $\mathcal{U}(\mathcal{H})$, and $V_{i}, V_{j}$ be irreducible $\mathbb{F} G$-modules. Choose $v_{i} \in V_{i}$ and $v_{j} \in V_{j}$ nonzero, and define

$$
S: V_{i} \rightarrow V_{j}, \quad S f:=\sum_{g \in G}\left\langle f, g v_{i}\right\rangle g v_{j}
$$

Then $S=0$ if $V_{i}$ and $V_{j}$ are not $\mathbb{F} G$-isomorphic. If $\sigma: V_{i} \rightarrow V_{j}$ is an $\mathbb{F} G$-isomorphism and $V_{i}$ is absolutely irreducible, then

$$
S f=\frac{|G|\left\|v_{i}\right\|^{2}}{\operatorname{dim}\left(V_{i}\right)\left\|\sigma v_{i}\right\|^{2}}\left\langle v_{j}, \sigma v_{i}\right\rangle \sigma f, \quad \forall f \in V_{i} .
$$

Proof: $\quad$ Observe that $S$ commutes with every $g \in G$, since

$$
g S(f)=g \sum_{h \in G}\left\langle f, h v_{i}\right\rangle h v_{j}=\sum_{h \in G}\left\langle g f, g h v_{i}\right\rangle g h v_{j}=S(g f),
$$


and so is an $\mathbb{F} G$-homomorphism. By Schur's Lemma, it follows that $S=0$ if $V_{i}$ and $V_{j}$ are not $\mathbb{F} G$-isomorphic.

Suppose now $V_{i}$ and $V_{j}$ are $\mathbb{F} G$-isomorphic, and write $S=\sum_{k} c_{k} \sigma_{k}$ where $\left\{\sigma_{k}\right\}$ is a basis for $\operatorname{Hom}_{\mathbb{F} G}\left(V_{i}, V_{j}\right)$ over $\mathbb{F}$. We then compute

$$
\begin{aligned}
\left\langle S v_{i}, \sigma_{r} v_{i}\right\rangle & =\left\langle\sum_{g \in G}\left\langle v_{i}, g v_{i}\right\rangle g v_{j}, \sigma_{r} v_{i}\right\rangle=\sum_{g \in G}\left\langle v_{i}, g v_{i}\right\rangle\left\langle g v_{j}, \sigma_{r} v_{i}\right\rangle \\
& =\sum_{g \in G}\left\langle g^{-1} v_{i}, v_{i}\right\rangle\left\langle v_{j}, \sigma_{r} g^{-1} v_{i}\right\rangle=\left\langle v_{j}, \sigma_{r} \sum_{g \in G}\left\langle v_{i}, g^{-1} v_{i}\right\rangle g^{-1} v_{i}\right\rangle .
\end{aligned}
$$

Since $V_{i}$ is irreducible, the $G$-orbit of $v_{i} \neq 0$ is an isometric tight frame for $V_{i}$, giving

$$
\sum_{g \in G}\left\langle v_{i}, g^{-1} v_{i}\right\rangle g^{-1} v_{i}=\frac{|G|}{\operatorname{dim}\left(V_{i}\right)}\left\|v_{i}\right\|^{2} v_{i}
$$

Hence the coefficients $\left\{c_{k}\right\}$ satisfy the invertible square linear system

$$
\sum_{k} c_{k}\left\langle\sigma_{k} v_{i}, \sigma_{r} v_{i}\right\rangle=\frac{|G|}{\operatorname{dim}\left(V_{i}\right)}\left\|v_{i}\right\|^{2}\left\langle v_{j}, \sigma_{r} v_{i}\right\rangle, \quad \forall r .
$$

This is invertible since the coefficient matrix is the Gramian of the vectors $\left\{\sigma_{k} v_{i}\right\}$ in $V_{j}$, which are linearly independent (since $\left\{\sigma_{k}\right\}$ is linearly independent and nonzero elements of $\operatorname{Hom}_{\mathbb{F} G}\left(V_{i}, V_{j}\right)$ are invertible). If $V_{i}$ is absolutely irreducible, then $\operatorname{Hom}_{\mathbb{F} G}\left(V_{i}, V_{j}\right)$ is one-dimensional, say spanned by $\sigma_{1}=\sigma$, and we can solve to obtain

$$
c_{1}=\frac{|G|\left\|v_{i}\right\|^{2}}{\operatorname{dim}\left(V_{i}\right)\left\|\sigma v_{i}\right\|^{2}}\left\langle v_{j}, \sigma v_{i}\right\rangle
$$

as required.

Theorem 6.18. Let $G$ be a finite group which acts on $\mathcal{H}$ as unitary transformations, and $\mathcal{H}=V_{1} \oplus V_{2} \oplus \cdots \oplus V_{k}$ an orthogonal direct sum of irreducible $\mathbb{F} G$-modules for which the summands that occur more than once are absolutely irreducible. If $v=v_{1}+v_{2}+\cdots+v_{k}$, $v_{i} \in V_{i}$, then $G v$ is an isometric tight frame for $\mathcal{H}$ if and only if

$$
\frac{\left\|v_{i}\right\|^{2}}{\left\|v_{j}\right\|^{2}}=\frac{\operatorname{dim}\left(V_{i}\right)}{\operatorname{dim}\left(V_{j}\right)}, \quad \forall i, j
$$

and in the case $V_{i}$ is $\mathbb{F} G$-isomorphic to $V_{j}, i \neq j$ via $\sigma: V_{i} \rightarrow V_{j}$ that

$$
\left\langle\sigma v_{i}, v_{j}\right\rangle=0
$$

Proof: $\quad$ By Lemma 6.7, necessary and sufficient conditions for $G v$ to be an isometric tight frame for $\mathcal{H}$ are (6.19), and, in the notation of Lemma 6.14, that $S v_{i}=0$. By Lemma 6.14, this last condition holds by virtue of the fact $S=0$, except in the case that there is an $\mathbb{F} G$-isomorphism $\sigma: V_{i} \rightarrow V_{j}$ between absolutely irreducibles, whence (6.15) gives

$$
S\left(v_{i}\right)=\frac{|G|\left\|v_{i}\right\|^{2}}{\operatorname{dim}\left(V_{i}\right)\left\|\sigma v_{i}\right\|^{2}}\left\langle v_{j}, \sigma v_{i}\right\rangle \sigma v_{i}=0 \quad \Longleftrightarrow \quad\left\langle\sigma v_{i}, v_{j}\right\rangle=0 .
$$


In particular, if isomorphic one-dimensional irreducibles occur in the orthogonal decomposition of $\mathcal{H}$, then no $G$-orbit can be a tight frame (since a 1 -dimensional irreducible is absolutely irreducible).

Next we use Theorem 6.18 to construct transitive tight frames of orthogonal polynomials of several variables which share symmetries of the weight. In each of our examples no irreducible occurs more than once, and so there is no need to check (6.20).

\section{Applications to orthogonal polynomials}

Here we apply our results to construct tight frames of orthogonal polynomials of several variables which share the symmetries of the weight.

Let $\Pi:=\Pi\left(\mathbb{R}^{d}\right)$ denote the polynomials in $d$ real variables, and $\Pi_{k}:=\Pi\left(\mathbb{R}^{d}\right)$ those of degree $\leq k$. The most general definition of orthogonal polynomials, see, [DX01], is in terms of a moment functional $\lambda: \Pi \rightarrow \mathbb{R}$. For simplicity, we suppose $\lambda$ is given by a suitable measure $\mu$, i.e., one with

$$
\lambda: \Pi \rightarrow \mathbb{R}: f \mapsto \int f d \mu<\infty
$$

and denote the corresponding inner product on polynomials by

$$
\left\langle f_{1}, f_{2}\right\rangle_{\mu}:=\int f_{1} f_{2} d \mu, \quad \forall f_{1}, f_{2} \in \Pi
$$

We say $f \in \Pi_{k}$ is an orthogonal polynomial with respect to the measure $\mu$ if

$$
\langle f, p\rangle_{\mu}=0, \quad \forall p \in \Pi_{k-1}
$$

and denote the Hilbert space of such orthogonal polynomials of degree $k$ by $\mathcal{V}_{k}(\mu)$.

Definition. The symmetry group of $\mu$ is

$$
\operatorname{Sym}(\mu):=\left\{g \in \operatorname{Aff}\left(\mathbb{R}^{d}\right): \int f \circ g d \mu=\int f d \mu, \forall f \in \Pi\right\},
$$

where $\operatorname{Aff}\left(\mathbb{R}^{d}\right)$ denotes the group of affine transformations on $\mathbb{R}^{d}$.

This acts on $\Pi$ via $g \cdot f:=f \circ g^{-1}$, with each $g \in \operatorname{Sym}(\mu)$ inducing a unitary transformation

$$
\left\langle g \cdot f_{1}, g \cdot f_{2}\right\rangle_{\mu}=\int\left(f_{1} \circ g^{-1}\right)\left(f_{2} \circ g^{-1}\right) d \mu=\int\left(f_{1} f_{2}\right) \circ g^{-1} d \mu=\int f_{1} f_{2} d \mu=\left\langle f_{1}, f_{2}\right\rangle_{\mu}
$$

which maps $\mathcal{V}_{k}(\mu)$ onto itself since $g \cdot \Pi_{k-1}=\Pi_{k-1}$, i.e., $\mathcal{V}_{k}(\mu)$ is a $G$-invariant subspace. More detail can be found in [W95]. 
Definition. Let $G$ be a subgroup of $\operatorname{Sym}(\mu)$. We say a tight frame $\Phi$ for $\mathcal{V}_{k}(\mu)$ has the symmetries $G$ of $\mu$ if it is $G$-invariant, i.e.,

$$
g \cdot \Phi=\Phi, \quad \forall g \in G,
$$

and when $G=\operatorname{Sym}(\mu)$ that it shares the symmetries of $\mu$.

This is equivalent to $\left.G \cdot\right|_{\mathcal{V}_{k}(\mu)} \subset \operatorname{Sym}(\Phi)$, where $\left.G \cdot\right|_{\mathcal{V}_{k}(\mu)}$ denotes the group of unitary transformations $\mathcal{V}_{k}(\mu) \rightarrow \mathcal{V}_{k}(\mu)$ given by $f \mapsto g \cdot f, g \in G$. We now consider examples of tight frames of Jacobi polynomials which share the symmetries of the weight.

Let $V$ be a set of $d+1$ affinely independent points in $\mathbb{R}^{d}$, i.e., the vertices of a $d$-simplex which we denote by $T$. Let $\xi=\left(\xi_{v}\right)_{v \in V}$ be the corresponding barycentric coordinates. For the (standard) triangle with vertices $0, e_{1}=(1,0), e_{2}=(0,1)$, these are

$$
\xi_{0}(x, y)=1-x-y, \quad \xi_{e_{1}}(x, y)=x, \quad \xi_{e_{2}}(x, y)=y .
$$

We will use standard multi-index notation for indices, so, for example,

$$
\xi^{\kappa}:=\prod_{v \in V} \xi_{v}^{\kappa_{v}}, \quad \kappa \in \mathbb{R}^{V}, \quad \beta !:=\prod_{v \in V} \beta_{v} !, \quad \beta \in \mathbb{Z}_{+}^{V} .
$$

For functions defined on $T$, we define an inner product by

$$
\langle f, g\rangle_{\mu}:=\int_{T} f g \xi^{\kappa}, \quad \kappa>-1,
$$

where the condition $\kappa_{v}>-1$ ensures the nonnegative weight $\xi^{\kappa}$ is integrable over the simplex $T$. The corresponding orthogonal polynomials are called the Jacobi polynomials for the simplex $T$ with weight $\xi^{\kappa}$, and Legendre polynomials when the weight is constant, i.e., $\xi^{\kappa}=1$.

The symmetry group of such a Jacobi measure is a group of affine maps which map $T$ onto $T$, which we represent as elements of $S_{V}$ the permutations on the vertices $V$ (since an affine map $\mathbb{R}^{d} \rightarrow \mathbb{R}^{d}$ is uniquely determined by its action on $d+1$ affinely independent points such as $V$ ). It is given by

$$
G:=\operatorname{Sym}(\mu)=\left\{g \in S_{V}: \kappa_{g v}=\kappa_{v}, \forall v \in V\right\} .
$$

A tight frame for $\mathcal{V}_{k}(\mu)$ which shares the symmetries of this weight was given in [PW02] (and [WX01]) and independently by [R99]. The structural form of this representation is

$$
f=\sum_{\substack{|\beta|=k \\ \beta \in \mathbb{Z}_{+}^{V}}} c_{\beta}^{\kappa}\left\langle f, p_{\beta}^{\kappa}\right\rangle p_{\beta}^{\kappa}, \quad \forall f \in \mathcal{V}_{k}(\mu),
$$

where $p_{\beta}^{\kappa}:=F(|\beta|+|\kappa|+d,-\beta, \kappa+1 ; \xi)$ with $F$ the Lauricella function, which is clearly $G$-invariant since $G$ permutes the $\xi_{v}$ with the same value of $\kappa_{v}$. These are not transitive 
tight frames for $k \geq 2$. For the Legendre polynomials on a triangle we determine whether or not a transitive tight frame exists.

For any measure $\mu$ an orthogonal polynomial $f \in \mathcal{V}_{k}(\mu)$ is uniquely determined by its leading term $f_{\uparrow}$, i.e., the homogeneous polynomial of degree $k$ for which $\operatorname{deg}\left(f-f_{\uparrow}\right)<k$, via

$$
f=f_{\uparrow}-P_{\Pi_{k-1}}\left(f_{\uparrow}\right), \quad P_{\Pi_{k-1}}:=\text { orthogonal projection onto } \Pi_{k-1}\left(\mathbb{R}^{s}\right) .
$$

Thus the space of orthogonal polynomials of (exact) degree $k$ has

$$
\operatorname{dim}\left(\mathcal{V}_{k}(\mu)\right)=\left(\begin{array}{c}
k+d-1 \\
d-1
\end{array}\right)
$$

Denote the Legendre polynomial with leading term $\left(\xi^{\beta}\right)_{\uparrow}, \beta \in \mathbb{Z}_{+}^{V},|\beta|=k$ by $p_{\xi^{\beta}}$. The group $G:=\operatorname{Sym}(\mu)=S_{V}$ of symmetries of the Legendre measure acts faithfully on the Legendre polynomials of degree $k \geq 1$, since the polynomials $p_{\xi_{v}^{k}}, v \in V$ are distinct and each element of $G$ permutes them in a different way. We normalise the Legendre inner product, so that corresponding norm has $\|1\|=1$.

Quadratic Legendre polynomials. Consider the 3-dimensional space of quadratic Legendre polynomials on the triangle with vertices $V=\{u, v, w\}$. Clearly the 1-dimensional subspace $V_{1}$ spanned by

$$
f_{1}:=p_{\xi_{u}^{2}+\xi_{v}^{2}+\xi_{w}^{2}}=p_{\xi_{u}^{2}}+p_{\xi_{v}^{2}}+p_{\xi_{w}^{2}}=\xi_{u}^{2}+\xi_{v}^{2}+\xi_{w}^{2}-\frac{1}{2}, \quad\left\|f_{1}\right\|^{2}=\frac{1}{60}
$$

is $G$-invariant ( $G$ fixes $f_{1}$ ), and its $G$-invariant orthogonal complement $V_{2}:=V_{1}^{\perp}$ is irreducible (since otherwise $G \approx S_{3}$ would be diagonalisable and hence abelian). Hence by Theorem 6.18 if we take a sum of components (with the appropriate norms) from each of these subspaces the $G$-orbit of the resulting vector $f$ will be a transitive tight frame. The orthogonal projection of $p_{\xi_{v}^{2}}$ onto $V_{2}$ is given by

$$
f_{2}:=P_{V_{2}}\left(p_{\xi_{v}^{2}}\right)=p_{\xi_{v}^{2}}-\frac{1}{3}\left(p_{\xi_{u}^{2}}+p_{\xi_{v}^{2}}+p_{\xi_{w}^{2}}\right), \quad\left\|f_{2}\right\|^{2}=\frac{1}{675} .
$$

Since $p_{\xi_{v}^{2}}=p_{\xi_{u}^{2}}+p_{\xi_{w}^{2}}-2 p_{\xi_{u} \xi_{w}}$, this can also be expressed as

$$
-f_{2}=p_{\xi_{u} \xi_{w}}+\frac{1}{6}\left(p_{\xi_{u}^{2}}+p_{\xi_{v}^{2}}+p_{\xi_{w}^{2}}\right)=P_{V_{2}}\left(p_{\xi_{u} \xi_{w}}\right)
$$

Hence the 3 vectors in the $G$-orbit of

$$
f:=\frac{f_{1}}{\left\|f_{1}\right\|} \frac{1}{\sqrt{3}} \pm \frac{f_{2}}{\left\|f_{2}\right\|} \sqrt{\frac{2}{3}}=(2 \sqrt{5} \mp 5 \sqrt{2}) p_{\xi_{u}^{2}+\xi_{v}^{2}+\xi_{w}^{2}} \pm 15 \sqrt{2} p_{\xi_{v}^{2}}
$$

form an orthonormal basis for the 3 -dimensional space $\mathcal{V}_{2}(\mu)$ which shares the symmetries of the Legendre measure. By contrast, the representation of (7.1) consists of 6 vectors with two different scaling factors. We can also write this $f$ in the form

$$
f=\left(2 \sqrt{5} \mp \frac{5}{2} \sqrt{2}\right) p_{\xi_{u}^{2}+\xi_{v}^{2}+\xi_{w}^{2}} \mp 15 \sqrt{2} p_{\xi_{u} \xi_{w}} .
$$


We now depict the quadratic $f$ whose orbit is an orthonormal basis, which is given by

$$
f=(2 \sqrt{5}-5 \sqrt{2})\left(\xi_{v}^{2}+\xi_{w}^{2}+\xi_{u}^{2}-\frac{1}{2}\right)+15 \sqrt{2}\left(\xi_{v}^{2}-\frac{4}{5} \xi_{v}+\frac{1}{10}\right)
$$
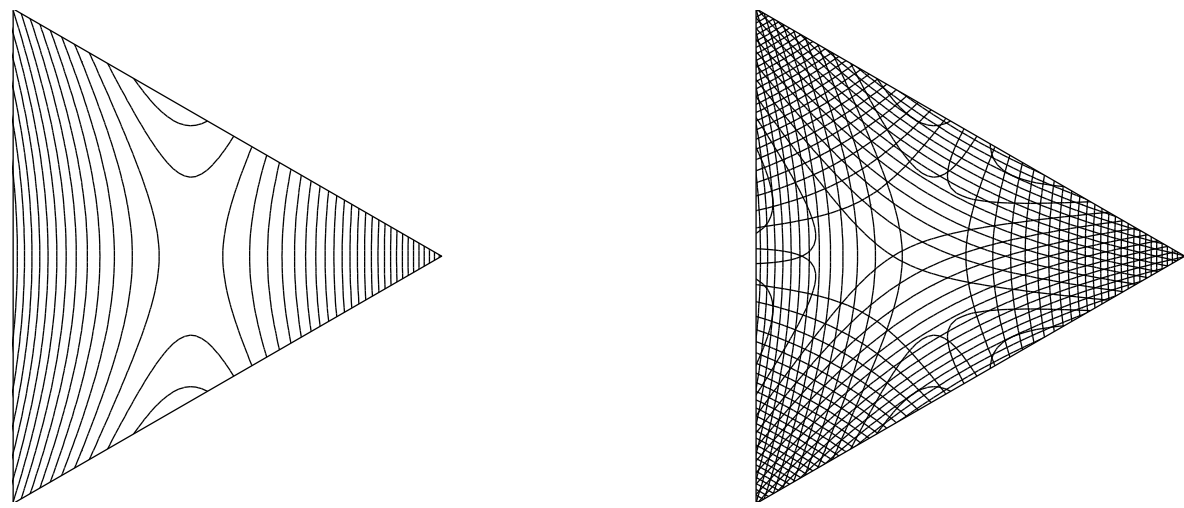

Fig. 1. Contour plots of $f$ and those of its orbit showing the triangular symmetry.

Cubic Legendre polynomials. Consider the cubic Legendre polynomials on a triangle. There is a 1 -dimensional $G$-invariant subspace (on which $G$ acts as the identity) given by

$$
f_{1}:=p_{\xi_{u} \xi_{v} \xi_{w}}, \quad\left\|f_{1}\right\|^{2}=\frac{1}{29400},
$$

and a 1-dimensional $G$-invariant subspace (on which $G$ acts as $C_{2}$ ) given by

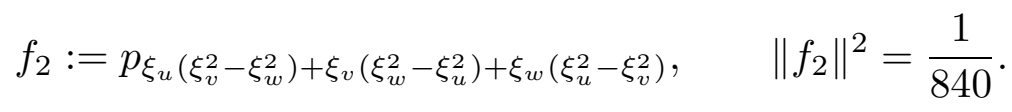

These spaces are orthogonal. The projection of $p_{\xi_{v}^{3}}$ onto the orthogonal complement of $f_{1}$ and $f_{2}$ is

$$
f_{3}:=p_{\xi_{v}^{3}}-p_{\xi_{u} \xi_{v} \xi_{w}}, \quad\left\|f_{3}\right\|^{2}=\frac{1}{5880},
$$

which has a 2 -dimensional $G$-orbit. Thus the $G$-orbit of any vector of the form

$$
f=c_{1} \frac{1}{\sqrt{4}} \frac{f_{1}}{\left\|f_{1}\right\|}+c_{2} \frac{1}{\sqrt{4}} \frac{f_{2}}{\left\|f_{2}\right\|}+c_{3} \frac{\sqrt{2}}{\sqrt{4}} \frac{f_{3}}{\left\|f_{3}\right\|}, \quad\left|c_{1}\right|=\left|c_{2}\right|=\left|c_{3}\right|=1
$$

is a transitive tight frame for the Legendre cubics.

Taking $c_{i}=1$, the orbit of $f$ is

$$
\left\{(35 \sqrt{6}-14 \sqrt{15}) f_{1}+c \sqrt{210} f_{2}+14 \sqrt{15} p_{\xi_{v}^{3}}: c \in\{-1,1\}, v \in V\right\},
$$

which is a transitive tight frame of 6 vectors for the 4-dimensional space $\mathcal{V}_{3}(\mu)$ which shares the symmetries of the Legendre measure. The representation of (7.1) consists of 10 vectors with three different scaling factors. 
Quartic Legendre polynomials. The quartics $\mathcal{V}_{4}(\mu)$ have two different $\mathbb{R} G$-isomorphic 1-dimensional $G$-invariant subspaces, given by

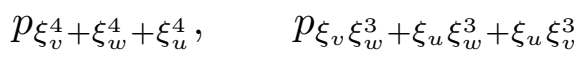

and so there can be no transitive tight frame for the quartics which shares the symmetries of the Legendre measure. Similarly, none exists for $\mathcal{V}_{5}(\mu)$ because of the $G$-invariant subspaces given by

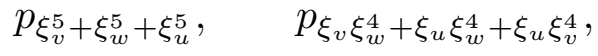

and none exists for $\mathcal{V}_{k}(\mu), k \geq 6$ since this space has dimension $k+1>6$ and a $G$-orbit has at most $|G|=6$ elements.

In the paper [Du87] the orthogonal polynomials on the hexagon are considered, i.e., those for $\mu$ the normalised Lebesgue integral over the hexagon $H$ with vertices

$$
\{(\sin (\pi j / 3), \cos (\pi j / 3)): j=1, \ldots, 6\},
$$

which has symmetry group $G=D_{6}$. The method given there is to split $\mathcal{V}_{k}(\mu)$ into the orthogonal direct sum of its homogeneous components $H\left(V_{j}\right)$ corresponding to different irreducible $G$-invariant subspaces $V_{j}$. (If $\mathcal{V}_{k}(\mu)=V_{1} \oplus V_{2} \oplus \cdots \oplus V_{k}$ is a decomposition of $\mathcal{V}_{k}(\mu)$ into a direct sum of irreducibles, the homogeneous component $H\left(V_{j}\right)$ is defined to be $\left.H\left(V_{j}\right)=\oplus_{V_{r} \approx V_{j}} V_{r}\right)$. Then for each $H\left(V_{j}\right)$ use Gram-Schmidt (in a clever way) to construct an orthonormal basis. For example, the quadratics have a 1-dimensional $G$-invariant subspace $V_{1}$ (on which $G$ acts as the identity) given by

$$
p_{2,0}:=x^{2}+y^{2}-\frac{5}{12}, \quad\left\|p_{2,0}\right\|^{2}=\frac{43}{720} .
$$

Its orthogonal complement $V_{2}:=V_{1}^{\perp}$ is a 2 -dimensional $G$-invariant subspace. This is further split $V_{2}=V_{2}^{+} \oplus V_{2}^{-}$into two 1-dimensional subspaces given by

$$
p_{2,+2}:=x^{2}-y^{2}+2 i x y, \quad p_{2,-2}:=x^{2}-y^{2}-2 i x y, \quad\left\|p_{2, \pm 2}\right\|=\frac{7}{30},
$$

which are nonisomorphic irreducible $C_{6}$-invariant subspaces, where $C_{6}$ is the rotation subgroup of $G=D_{6}$. The resulting orthogonal basis $\left\{p_{2,0}, p_{2,+2}, p_{2,-2}\right\}$ is therefore invariant under the rotations of the hexagon, with its first element also fixed by all symmetries of the hexagon. By Theorem 6.18, the $G$-orbit of any vector of the form

$$
f=\frac{f_{1}}{\left\|f_{1}\right\|} \frac{1}{\sqrt{3}}+\frac{f_{2}}{\left\|f_{2}\right\|} \frac{\sqrt{2}}{\sqrt{3}}, \quad f_{1} \in V_{1}, \quad f_{2} \in V_{2}
$$

is a transitive tight frame for the quadratics which shares the symmetries of the hexagon. The size of this orbit divides $|G|=12$. For the choice $f=x^{2}-5 / 24$, it consists of three vectors

$$
G f=\left\{x^{2}-\frac{5}{24},\left(\frac{1}{2} x-\frac{\sqrt{3}}{2} y\right)^{2}-\frac{5}{24},\left(\frac{1}{2} x+\frac{\sqrt{3}}{2} y\right)^{2}-\frac{5}{24}\right\}, \quad\|f\|^{2}=\frac{127}{2880},
$$


giving an orthonormal basis for $\mathcal{V}_{2}(\mu)$ which shares all the symmetries of the hexagon.
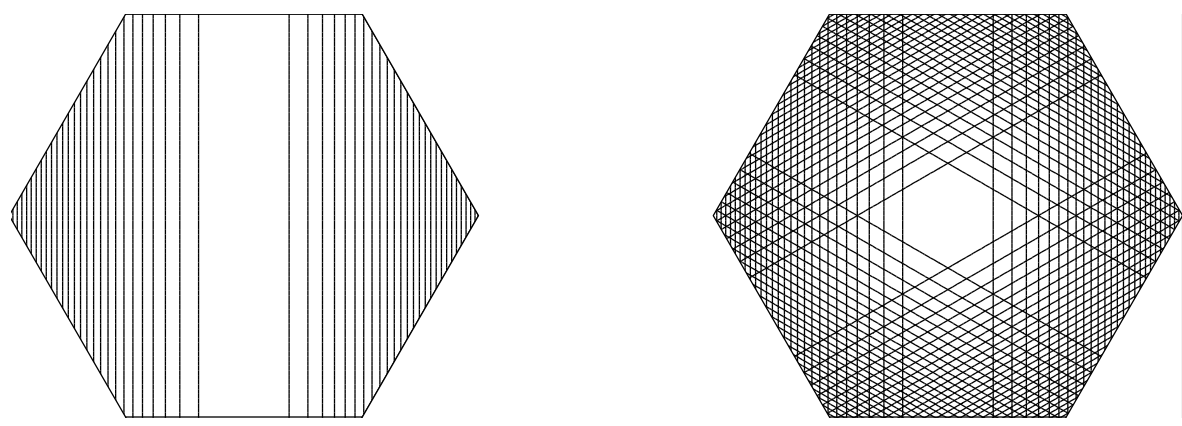

Fig. 2. Contour plots of $f$ and those of its orbit showing the hexagonal symmetry.

As a final examples, consider the 3-dimensional space of quadratic Legendre polynomials on the square $[-1,1]^{2} \subset \mathbb{R}^{2}$. This has symmetry group $D_{4}$. It is possible to construct a transitive tight frame of 4 vectors for this space. The orthogonal decomposition into homogeneous components consists of three 1-dimensional subspaces given by

$$
x^{2}+y^{2}-\frac{2}{3}, \quad x^{2}-y^{2}, \quad x y, \quad\|\phi\|^{2}=\frac{8}{45}, \frac{8}{45}, \frac{1}{9} .
$$

On the first $D_{4}$ acts as the identity, and on the others as $C_{2}$. The last two are not isomorphic irreducibles since the symmetry $(x, y) \mapsto(y, x)$ fixes $x y$ but not $x^{2}-y^{2}$. Hence taking any sum of vectors of equal length from these subspaces gives a vector $\phi$ whose orbit is a transitive tight frame, e.g.,

$$
\phi:=\frac{\sqrt{5}}{2}\left(x^{2}+y^{2}-2 / 3\right)+\frac{\sqrt{5}}{2}\left(x^{2}-y^{2}\right)+\sqrt{2} x y=\sqrt{5}\left(x^{2}-\frac{1}{3}\right)+\sqrt{2} x y
$$

gives the transitive tight frame

$$
D_{4} \phi=\left\{\sqrt{5}\left(x^{2}-\frac{1}{3}\right) \pm \sqrt{2} x y, \sqrt{5}\left(y^{2}-\frac{1}{3}\right) \pm \sqrt{2} x y\right\}, \quad\|\phi\|^{2}=\frac{2}{3} .
$$

which clearly shares the symmetries of the square. These are hyperbolic paraboloids since

$$
\phi=-\frac{\sqrt{5}}{3}+\frac{1}{2}\left\{(\sqrt{5}+\sqrt{7}) \frac{\left(\frac{\sqrt{5}+\sqrt{7}}{\sqrt{2}} x+y\right)^{2}}{\frac{(\sqrt{5}+\sqrt{7})^{2}}{2}+1}-(\sqrt{7}-\sqrt{5}) \frac{\left(\frac{\sqrt{5}-\sqrt{7}}{\sqrt{2}} x+y\right)^{2}}{\frac{(\sqrt{5}-\sqrt{7})^{2}}{2}+1}\right\}
$$
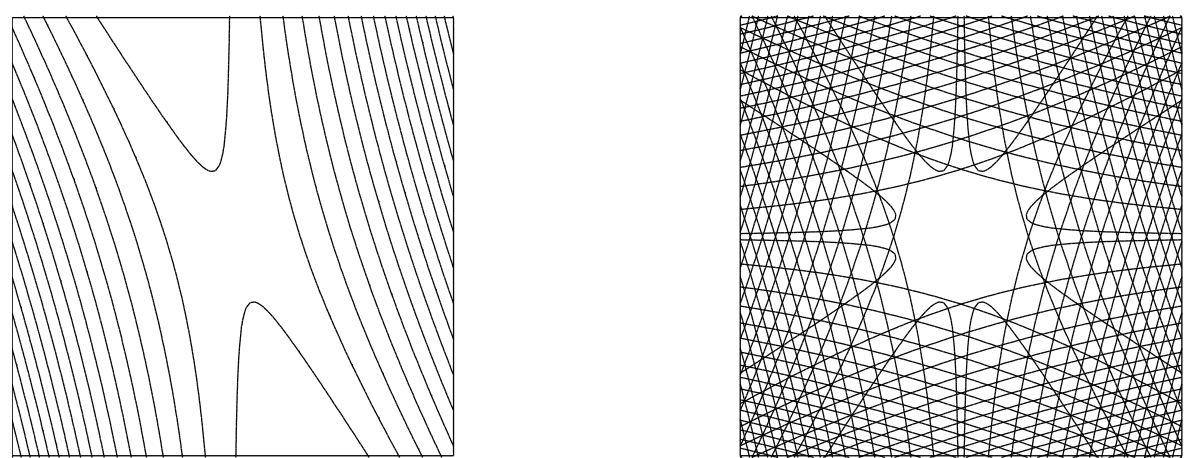

Fig. 3. Contour plots of $\phi$ and those of its orbit showing the square symmetry. 


\section{Acknowledgements}

We would like to thank Charles Leedham-Green for help with the representation theory and Eamonn O'Brien for answering many questions.

\section{References}

[AG93] N. I. Akhiezer and I. M. Glazman, "Theory of linear operators in Hilbert space", Dover, New York, 1993.

[BF03] J.J. Benedetto and M. Fickus, "Finite Normalized Tight Frames", Adv. Comp. Math.; 18, 2003, 357-385.

[B98] C. Blatter, "Wavelets - a primer", Friedr. Vieweg \& Sohn, Braunschweig, 1998.

[BG71] C. T. Benson and L. C. Grove, "Finite reflection groups", Bogden \& Quigley, New York, 1971.

[BE03] H. Bölcskei and Y. C. Eldar, Geometrically uniform frames, IEEE Trans. Inform. Theory 49 no. 4 (2003), 993-1006.

[CFKLT03] P. G. Casazza, M. C. Fickus, J. Kovačević, M. T. Leon, and J. C. Tremain, A physical interpretation for finite tight frames, Preprint, 2003.

[CK01] P. G. Casazza and J. Kovačević, Uniform tight frames with erasures, Preprint, 2001.

[D92] I. Daubechies, "Ten Lectures on Wavelets", CBMS Conf. Series in Appl. Math., vol. 61, SIAM, Philadelphia, 1992.

[Da79] P. J. Davis, "Circulant matrices", Wiley, New York, 1979.

[DS52] R. J. Duffin and A. C. Schaeffer, A Class of Nonharmonic Fourier Series, Trans. Amer. Math. Soc. 72(2) (1952), 341-366.

[Du87] C. F. Dunkl, Orthogonal polynomials on the hexagon, SIAM J. Appl. Math. 47(2) (1987), 343-351.

[DX01] C. F. Dunkl and Y. Xu, "Orthogonal polynomials of several variables", Cambridge University Press, Cambridge, 2001.

[E02] Y. C. Eldar, Least-squares inner product shaping, Linear Algebra Appl. 348 no. 1-3 (2002), 153-174.

[F01] M. Fickus, "Finite normalized tight frames and spherical equidistribution", dissertation, University of Maryland, 2001.

[Fol95] G. B. Folland, "A course in abstract harmonic analysis", CRC Press, Boca Raton, 1995.

[For91] G. D. Forney, Geometrically uniform codes, IEEE Trans. Inform. Theory 37(5) (1991), 1241-1260.

[GKK01] V. K. Goyal, J. Kovačević, and J. A. Kelner, Quantized Frame Expansions with Erasures, Appl. Comput. Harmonic Anal. 10 (2001), 203-233.

[GVT98] V. K. Goyal, M. Vetterli, and N. T. Thao, Quantized overcomplete expansions in $\mathbb{R}^{N}$ : analysis, synthesis, and algorithms, IEEE Trans. Inform. Theory 44(1) (1998), $16-31$.

[G97] L. C. Grove, "Groups and Characters", John Wiley and Sons, New York, 1997.

[HJ85] R. A. Horn and C. R. Johnson, "Matrix analysis", Cambridge University Press, Cambridge, 1985. 
[JL93] G. James and M. Liebeck, "Representations and Characters of Groups", Cambridge University Press, Cambridge, 1993.

[PW02] I. Peng and S. Waldron, Signed frames and Hadamard products of Gram matrices, Linear Algebra Appl. 347 no. 1-3 (2002), 131-157.

[RW02] R. Reams and S. Waldron, Isometric tight frames, Electron. J. Linear Algebra 9 (2002), 122-128.

[R99] H. Rosengren, Multivariable orthogonal polynomials and coupling coefficients for discrete series representations, SIAM J. Math. Anal. 30 (1999), 232-272.

[Ru62] W. Rudin, "Fourier Analysis on Groups", Interscience, Netherlands, 1960.

[VW03] R. Vale and S Waldron, The vertices of the Platonic Solids are Tight Frames, Preprint, 2003.

[W95] S Waldron, Symmetries of linear functionals, in "Approximation Theory VIII, Vol. 1: Approximation and Interpolation" (Charles K. Chui and Larry L. Schumaker Eds.), pp. 541-550, World Scientific Publishing Co., Inc., Singapore, 1995.

[WX01] S. Waldron and Y. Xu, Tight frames of Jacobi polynomials on a simplex, Preprint, 2001.

[Sl68] D. Slepian, Group codes for the Gaussian channel, Bell System Tech. J. 47 (1968), 575-602.

[Z01] G. Zimmermann, Normalized tight frames in finite dimensions, in "Recent Progress in Multivariate Approximation, ISNM 137" (W. Haussmann and K. Jetter Eds.), pp. 249-252, Birkhäuser, Basel, 2001. 